\title{
Adaptive mixture methods based on Bregman divergences
}

\author{
Mehmet A. Donmez ${ }^{a}$, Huseyin A. Inan ${ }^{a}$, Suleyman S. Kozat ${ }^{\mathrm{b}, *}$ \\ a Department of Electrical and Computer Engineering, Koc University, Istanbul, Turkey \\ ${ }^{\mathrm{b}}$ Department of Electrical and Electronics Engineering, Bilkent University, Ankara 06800, Turkey
}

\section{A R T I C L E I N F O}

\section{Article history:}

Available online 26 September 2012

\section{Keywords:}

Adaptive mixture

Bregman divergence

Affine mixture

Multiplicative update

\begin{abstract}
A B S T R A C T
We investigate adaptive mixture methods that linearly combine outputs of $m$ constituent filters running in parallel to model a desired signal. We use Bregman divergences and obtain certain multiplicative updates to train the linear combination weights under an affine constraint or without any constraints. We use unnormalized relative entropy and relative entropy to define two different Bregman divergences that produce an unnormalized exponentiated gradient update and a normalized exponentiated gradient update on the mixture weights, respectively. We then carry out the mean and the mean-square transient analysis of these adaptive algorithms when they are used to combine outputs of $m$ constituent filters. We illustrate the accuracy of our results and demonstrate the effectiveness of these updates for sparse mixture systems.
\end{abstract}

(c) 2012 Published by Elsevier Inc.

\section{Introduction}

In this paper, we study adaptive mixture methods based on Bregman divergences [1,2] that combine outputs of $m$ constituent filters running in parallel on the same task. The overall system has two stages [3-8]. The first stage contains adaptive filters running in parallel to model a desired signal. The outputs of these adaptive filters are then linearly combined to produce the final output of the overall system in the second stage. We use Bregman divergences and obtain certain multiplicative updates $[9,2,10]$ to train these linear combination weights under an affine constraint [11] or without any constraints [12]. We use unnormalized [2] and normalized relative entropy [9] to define two different Bregman divergences that produce the unnormalized exponentiated gradient update (EGU) and the exponentiated gradient update (EG) on the mixture weights [9], respectively. We then perform the mean and the mean-square transient analysis of these adaptive mixtures when they are used to combine outputs of $m$ constituent filters. We emphasize that to the best of our knowledge, this is the first mean and mean-square transient analysis of the EGU algorithm and the EG algorithm in the mixture framework (which naturally covers the classical framework also $[13,14]$ ). We illustrate the accuracy of our results through simulations in different configurations and demonstrate advantages of the introduced algorithms for sparse mixture systems.

\footnotetext{
* Corresponding author.

E-mail addresses: mdonmez@ku.edu.tr (M.A. Donmez), huseyin.inan@boun.edu.tr (H.A. Inan), kozat@ee.bilkent.edu.tr (S.S. Kozat).
}

Adaptive mixture methods are utilized in a wide range of signal processing applications in order to improve the steady-state and/or convergence performance over the constituent filters as well as to deal with the limitations of different type of adaptive filters, or to fight against the lack of information that would be necessary to optimally adjust their parameters $[11,12,15]$. An adaptive convexly constrained mixture of two filters is studied in [15], where the convex combination is shown to be "universal" such that the combination performs at least as well as its best constituent filter in the steady state [15]. The transient analysis of this adaptive convex combination is studied in [16], where the time evolution of the mean and variance of the mixture weights is provided. In similar lines, an affinely constrained mixture of adaptive filters using a stochastic gradient update is introduced in [11]. The steady-state mean-square error (MSE) of this affinely constrained mixture is shown to outperform the steady-state MSE of the best constituent filter in the mixture under certain conditions [11]. The transient analysis of this affinely constrained mixture for $m$ constituent filters is carried out in [17]. The general linear mixture framework as well as the steady-state performances of different mixture configurations are studied in [12].

In this paper, we use Bregman divergences to derive multiplicative updates on the mixture weights. We use the unnormalized relative entropy and the relative entropy as distance measures and obtain the EGU algorithm and the EG algorithm to update the combination weights under an affine constraint or without any constraints. We then carry out the mean and the mean-square transient analysis of these adaptive mixtures when they are used to combine $m$ constituent filters. We point out that the EG algorithm is widely used in sequential learning theory [18] and minimizes an approximate final estimation error while penalizing the 


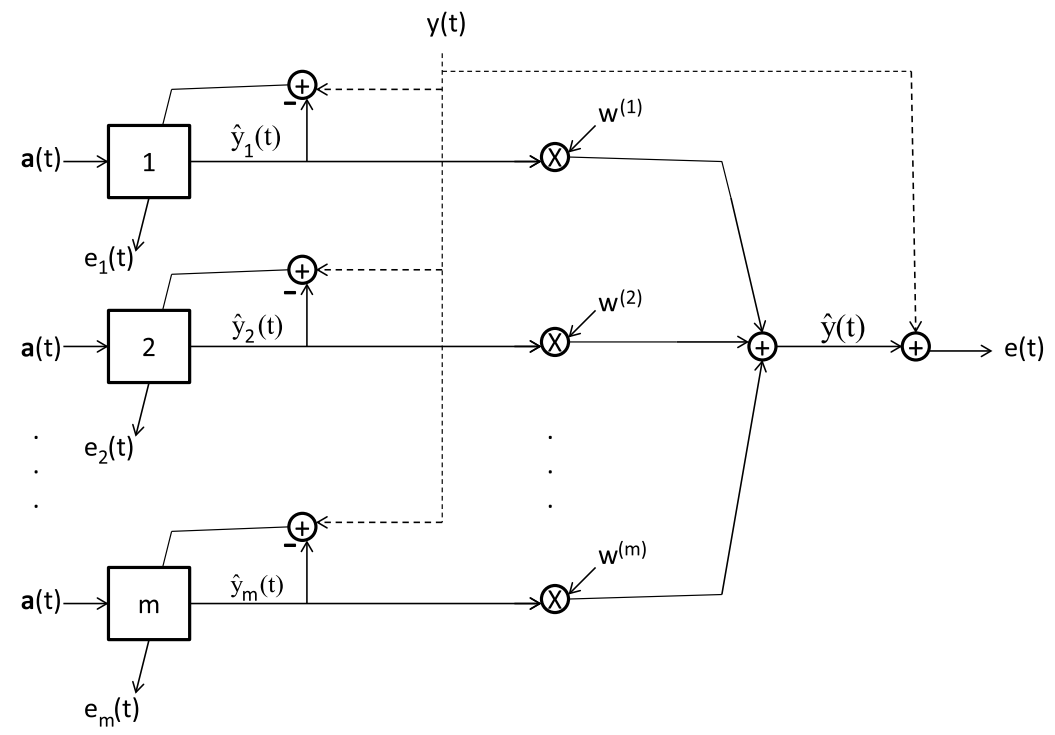

Fig. 1. A linear mixture of outputs of $m$ adaptive filters.

distance between the new and the old filter weights. In network and acoustic echo cancellation applications, the EG algorithm is shown to converge faster than the LMS algorithm [14,19] when the system impulse response is sparse [13]. Similarly, in our simulations, we observe that using the EG algorithm to train the mixture weights yields increased convergence speed compared to using the LMS algorithm to train the mixture weights $[11,12]$ when the combination favors only a few of the constituent filters in the steady state, i.e., when the final steady-state combination vector is sparse. We also observe that the EGU algorithm and the LMS algorithm show similar performance when they are used to train the mixture weights even if the final steady-state mixture is sparse. In this sense, we emphasize that we do not force the system to be sparse in order to make sure that the EG algorithm performs better than the LMS algorithm. However, if the final steady-state vector is sparse, than the EG could increase the convergence speed.

To summarize, the main contributions of this paper are as follows:

- We use Bregman divergences to derive multiplicative updates on affinely constrained and unconstrained mixture weights adaptively combining outputs of $m$ constituent filters.

- We use the unnormalized relative entropy and the relative entropy to define two different Bregman divergences that produce the EGU algorithm and the EG algorithm to update the affinely constrained and unconstrained mixture weights.

- We perform the mean and the mean-square transient analysis of the affinely constrained and unconstrained mixtures using the EGU algorithm and the EG algorithm.

The organization of the paper is as follows. In Section 2, we first describe the mixture framework. In Section 3, we study the affinely constrained and unconstrained mixture methods updated with the EGU algorithm and the EG algorithm. In Section 4, we first perform the transient analysis of the affinely constrained mixtures and then continue with the transient analysis of the unconstrained mixtures. Finally, in Section 5, we perform simulations to show the accuracy of our results and to compare performances of the different adaptive mixture methods. The paper concludes with certain remarks in Section 6.

\section{System description}

\subsection{Notation}

In this paper, all vectors are column vectors and represented by boldface lowercase letters. Matrices are represented by boldface capital letters. For presentation purposes, we work only with real data. Given a vector $\boldsymbol{w}, w^{(i)}$ denotes the $i$ th individual entry of $\boldsymbol{w}, \boldsymbol{w}^{T}$ is the transpose of $\boldsymbol{w},\|\boldsymbol{w}\|_{1} \triangleq \sum_{i}\left|w^{(i)}\right|$ is the $l_{1}$ norm; $\|\boldsymbol{w}\| \triangleq \sqrt{\boldsymbol{w}^{T} \boldsymbol{w}}$ is the $l_{2}$ norm. For a matrix $\boldsymbol{W}, \operatorname{tr}(\boldsymbol{W})$ is the trace. For a vector $\boldsymbol{w}, \operatorname{diag}(\boldsymbol{w})$ represents a diagonal matrix formed using the entries of $\boldsymbol{w}$. For a matrix $\boldsymbol{W}, \operatorname{diag}(\boldsymbol{W})$ represents a column vector that contains the diagonal entries of $\boldsymbol{W}$. For two vectors $\boldsymbol{v}_{1}$ and $\boldsymbol{v}_{2}$, we define the concatenation $\left[\boldsymbol{v}_{1} ; \boldsymbol{v}_{2}\right] \triangleq\left[\boldsymbol{v}_{1}^{T} \boldsymbol{v}_{2}^{T}\right]^{T}$. For a random variable $v, \bar{v}$ is the expected value. For a random vector $\boldsymbol{v}$ (or a random matrix $\boldsymbol{V}$ ), $\overline{\boldsymbol{v}}$ (or $\overline{\boldsymbol{V}}$ ) represents the expected value of each entry. Vectors (or matrices) $\mathbf{1}$ and $\mathbf{0}$, with an abuse of notation, denote vectors (or matrices) of all ones or zeros, respectively, where the size of the vector (or the matrix) is understood from the context.

\subsection{System description}

The framework that we study has two stages. In the first stage, we have $m$ adaptive filters producing outputs $\hat{y}_{i}(t), i=1, \ldots, m$, running in parallel to model a desired signal $y(t)$ as seen in Fig. 1. Here, $\boldsymbol{a}(t)$ is generated from a zero mean stochastic process and $y(t)$ is generated from a zero-mean stationary stochastic process. The second stage is the mixture stage, where the outputs of the first stage filters are combined to improve the steadystate and/or the transient performance over the constituent filters. We linearly combine the outputs of the first stage filters to produce the final output as $\hat{y}(t)=\boldsymbol{w}^{T}(t) \boldsymbol{x}(t)$, where $\boldsymbol{x}(t) \triangleq$ $\left[\hat{y}_{1}(t), \ldots, \hat{y}_{m}(t)\right]^{T}$ and train the mixture weights using multiplicative updates (or exponentiated gradient updates) [2]. We point out that in order to satisfy the constraints and derive the multiplicative updates $[9,20]$, we use reparametrization of the mixture weights as $\boldsymbol{w}(t)=\boldsymbol{f}(\lambda(t))$ and perform the update on $\lambda(t)$ as

$\lambda(t+1)=\arg \min _{\lambda}\left\{d(\lambda, \lambda(t))+\mu l\left(y(t), \boldsymbol{f}^{T}(\lambda) \boldsymbol{x}(t)\right)\right\}$,

where $\mu$ is the learning rate of the update, $d(\cdot, \cdot)$ is an appropriate distance measure and $l(\cdot, \cdot)$ is the instantaneous loss. We 
emphasize that in (1), the updated vector $\lambda$ is forced to be close to the present vector $\lambda(t)$ by $d(\lambda(t+1), \lambda(t))$, while trying to accurately model the current data by $l\left(y(t), \boldsymbol{f}^{T}(\lambda) \boldsymbol{x}(t)\right)$. However, instead of directly minimizing (1), a linearized version of (1)

$$
\begin{aligned}
\lambda(t+1)= & \arg \min _{\lambda}\left\{d(\lambda, \lambda(t))+l\left(y(t), \boldsymbol{f}^{T}(\lambda(t)) \boldsymbol{x}(t)\right)\right. \\
& \left.+\left.\mu \nabla_{\lambda} l\left(y(t), \boldsymbol{f}^{T}(\lambda) \boldsymbol{x}(t)\right)^{T}\right|_{\lambda=\lambda(t)}(\lambda-\lambda(t))\right\}
\end{aligned}
$$

is minimized to get the desired update. As an example, if we use the $l_{2}$-norm as the distance measure, i.e., $d(\lambda, \lambda(t))=\|\lambda-\lambda(t)\|^{2}$, and the square error as the instantaneous loss, i.e., $l(y(t)$, $\left.\boldsymbol{f}^{T}(\lambda) \boldsymbol{x}(t)\right)=\left[y(t)-\boldsymbol{f}^{T}(\boldsymbol{\lambda}) \boldsymbol{x}(t)\right]^{2}$ with $\boldsymbol{f}(\lambda)=\lambda$, then we get the stochastic gradient update on $\boldsymbol{w}(t)$, i.e.,

$\boldsymbol{w}(t+1)=\boldsymbol{w}(t)+\mu e(t) \boldsymbol{x}(t)$,

in (2).

In the next section, we use the unnormalized relative entropy

$d_{1}(\lambda, \lambda(t))=\left\{\sum_{i=1}^{m}\left[\lambda^{(i)} \ln \left(\frac{\lambda^{(i)}}{\lambda^{(i)}(t)}\right)+\lambda^{(i)}(t)-\lambda^{(i)}\right]\right\}$

for positively constrained $\lambda$ and $\lambda(t), \lambda \in \mathbb{R}_{+}^{m}, \lambda(t) \in \mathbb{R}_{+}^{m}$, and the relative entropy

$d_{2}(\lambda, \lambda(t))=\left\{\sum_{i=1}^{m}\left[\lambda^{(i)} \ln \left(\frac{\lambda^{(i)}}{\lambda^{(i)}(t)}\right)\right]\right\}$,

where $\lambda$ is constrained to be in an extended simplex such that $\lambda^{(i)} \geqslant 0, \sum_{k=1}^{m} \lambda^{(i)}=u$ for some $u \geqslant 1$ as the distance measures, with appropriately selected $\boldsymbol{f}(\cdot)$ to derive updates on mixture weights under different constraints. We first investigate affinely constrained mixture of $m$ adaptive filters, and then continue with the unconstrained mixture using (3) and (4) as the distance measures.

\section{Adaptive mixture algorithms}

In this section, we investigate affinely constrained and unconstrained mixtures updated with the EGU algorithm and the EG algorithm.

\subsection{Affinely constrained mixture}

When an affine constraint is imposed on the mixture such that $\boldsymbol{w}^{T}(t) \mathbf{1}=1$, we get

$\hat{y}(t)=\boldsymbol{w}(t)^{T} \boldsymbol{x}(t)$,

$e(t)=y(t)-\hat{y}(t)$,

$w^{(i)}(t)=\lambda^{(i)}(t), \quad i=1, \ldots, m-1$,

$w^{(m)}(t)=1-\sum_{i=1}^{m-1} \lambda^{(i)}(t)$,

where the $(m-1)$-dimensional vector $\lambda(t) \triangleq\left[\lambda^{(1)}(t), \ldots, \lambda^{(m-1)}(t)\right]^{T}$ is the unconstrained weight vector, i.e., $\lambda(t) \in \mathbb{R}^{m-1}$. Using $\lambda(t)$ as the unconstrained weight vector, the error can be written as $e(t)=\left[y(t)-\hat{y}_{m}(t)\right]-\lambda^{T}(t) \delta(t)$, where $\delta(t) \triangleq\left[\hat{y}_{1}(t)-\hat{y}_{m}(t), \ldots\right.$, $\left.\hat{y}_{m-1}(t)-\hat{y}_{m}(t)\right]^{T}$. To be able to derive a multiplicative update on $\lambda(t)$, we use

$\lambda(t)=\lambda_{1}(t)-\lambda_{2}(t)$, where $\lambda_{1}(t)$ and $\lambda_{2}(t)$ are constrained to be nonnegative, i.e., $\lambda_{i}(t) \in \mathbb{R}_{+}^{m-1}, i=1,2$. After we collect nonnegative weights in $\lambda_{a}(t)=\left[\lambda_{1}(t) ; \lambda_{2}(t)\right]$, we define a function of loss $e(t)$ as

$l_{\mathrm{a}}\left(\lambda_{a}(t)\right) \triangleq e^{2}(t)$

and update positively constrained $\lambda_{a}(t)$ as follows.

\subsubsection{Unnormalized relative entropy}

Using the unconstrained relative entropy as the distance measure, we get

$$
\begin{aligned}
\lambda_{a}(t+1)= & \arg \min _{\lambda}\left\{\sum_{i=1}^{2(m-1)}\left[\lambda^{(i)} \ln \left(\frac{\lambda^{(i)}}{\lambda_{a}^{(i)}(t)}\right)+\lambda_{a}^{(i)}(t)-\lambda^{(i)}\right]\right. \\
& \left.+\mu\left[l_{\mathrm{a}}\left(\lambda_{a}(t)\right)+\left.\nabla_{\lambda} l_{\mathrm{a}}(\lambda)^{T}\right|_{\lambda=\lambda_{a}(t)}\left(\lambda-\lambda_{a}(t)\right)\right]\right\} .
\end{aligned}
$$

After some algebra this yields

$$
\begin{aligned}
& \lambda_{a}^{(i)}(t+1)=\lambda_{a}^{(i)}(t) \exp \left\{\mu e(t)\left(\hat{y}_{i}(t)-\hat{y}_{m}(t)\right)\right\}, \\
& \quad i=1, \ldots, m-1, \\
& \lambda_{a}^{(i)}(t+1)=\lambda_{a}^{(i)}(t) \exp \left\{-\mu e(t)\left(\hat{y}_{i-m+1}(t)-\hat{y}_{m}(t)\right)\right\}, \\
& \quad i=m, \ldots, 2(m-1),
\end{aligned}
$$

providing the multiplicative updates on $\lambda_{1}(t)$ and $\lambda_{2}(t)$.

\subsubsection{Relative entropy}

Using the relative entropy as the distance measure, we get

$$
\begin{aligned}
\lambda_{a}(t+1)= & \arg \min _{\lambda}\left\{\sum_{i=1}^{2(m-1)}\left[\lambda^{(i)} \ln \left(\frac{\lambda^{(i)}}{\lambda_{a}^{(i)}(t)}\right)+\gamma\left(u-\mathbf{1}^{T} \lambda\right)\right]\right. \\
& \left.+\mu\left[l_{\mathrm{a}}\left(\lambda_{a}(t)\right)+\left.\nabla_{\lambda} l_{\mathrm{a}}(\lambda)^{T}\right|_{\lambda=\lambda_{a}(t)}\left(\lambda-\lambda_{a}(t)\right)\right]\right\},
\end{aligned}
$$

where $\gamma$ is the Lagrange multiplier. This yields

$$
\begin{aligned}
\lambda_{a}^{(i)}(t+1)= & u\left(\lambda_{a}^{(i)}(t) \exp \left\{\mu e(t)\left(\hat{y}_{i}(t)-\hat{y}_{m}(t)\right)\right\}\right) \\
& \times\left(\sum _ { k = 1 } ^ { m - 1 } \left[\lambda_{a}^{(k)}(t) \exp \left\{\mu e(t)\left(\hat{y}_{k}(t)-\hat{y}_{m}(t)\right)\right\}\right.\right. \\
& \left.\left.+\lambda_{a}^{(k+m-1)}(t) \exp \left\{-\mu e(t)\left(\hat{y}_{k}(t)-\hat{y}_{m}(t)\right)\right\}\right]\right)^{-1}, \\
i=1, \ldots, m-1, & \\
\lambda_{a}^{(i)}(t+1)= & u\left(\lambda_{a}^{(i)}(t) \exp \left\{-\mu e(t)\left(\hat{y}_{i-m+1}(t)-\hat{y}_{m}(t)\right)\right\}\right) \\
& \times\left(\sum _ { k = 1 } ^ { m - 1 } \left[\lambda_{a}^{(k)}(t) \exp \left\{\mu e(t)\left(\hat{y}_{k}(t)-\hat{y}_{m}(t)\right)\right\}\right.\right. \\
& \left.\left.+\lambda_{a}^{(k+m-1)}(t) \exp \left\{-\mu e(t)\left(\hat{y}_{k}(t)-\hat{y}_{m}(t)\right)\right\}\right]\right)^{-1},
\end{aligned}
$$$$
i=m, \ldots, 2(m-1),
$$

providing the multiplicative updates on $\lambda_{a}(t)$.

\subsection{Unconstrained mixture}

Without any constraints on the combination weights, the mixture stage can be written as 
$\hat{y}(t)=\boldsymbol{w}^{T}(t) \boldsymbol{x}(t)$

$e(t)=y(t)-\hat{y}(t)$,

where $\boldsymbol{w}(t) \in \mathbb{R}^{m}$. To be able to derive a multiplicative update, we use a change of variables,

$\boldsymbol{w}(t)=\boldsymbol{w}_{1}(t)-\boldsymbol{w}_{2}(t)$,

where $\boldsymbol{w}_{1}(t)$ and $\boldsymbol{w}_{2}(t)$ are constrained to be nonnegative, i.e., $\boldsymbol{w}_{i}(t) \in \mathbb{R}_{+}^{m}, i=1,2$. We then collect the nonnegative weights $\boldsymbol{w}_{a}(t)=\left[\boldsymbol{w}_{1}(t) ; \boldsymbol{w}_{2}(t)\right]$ and define a function of the loss $e(t)$ as

$l_{\mathrm{u}}\left(\boldsymbol{w}_{a}(t)\right) \triangleq e^{2}(t)$

\subsubsection{Unnormalized relative entropy}

Defining cost function similar to (4) and minimizing it with respect to $\boldsymbol{w}$ yields

$w_{a}^{(i)}(t+1)=w_{a}^{(i)}(t) \exp \left\{\mu e(t) \hat{y}_{i}(t)\right\}, \quad i=1, \ldots, m$,

$w_{a}^{(i)}(t+1)=w_{a}^{(i)}(t) \exp \left\{-\mu e(t) \hat{y}_{i-m}(t)\right\}, \quad i=m+1, \ldots, 2 m$,

providing the multiplicative update on $\boldsymbol{w}_{a}(t)$.

\subsubsection{Relative entropy}

Using the relative entropy under the simplex constraint on $\boldsymbol{w}$, we get the updates

$$
\begin{aligned}
& w_{a}^{(i)}(t+1)=u \frac{w_{a}^{(i)}(t) \exp \left\{\mu e(t) \hat{y}_{i}(t)\right\}}{\sum_{k=1}^{m}\left[w_{a}^{(k)}(t) \exp \left\{\mu e(t) \hat{y}_{k}(t)\right\}+w_{a}^{(k+m)}(t) \exp \left\{-\mu e(t) \hat{y}_{k}(t)\right\}\right]}, \\
& \quad i=1, \ldots, m, \\
& w_{a}^{(i)}(t+1)=u \frac{w_{a}^{(i)}(t) \exp \left\{-\mu e(t) \hat{y}_{i-m}(t)\right\}}{\sum_{k=1}^{m}\left[w_{a}^{(k)}(t) \exp \left\{\mu e(t) \hat{y}_{k}(t)\right\}+w_{a}^{(k+m)}(t) \exp \left\{-\mu e(t) \hat{y}_{k}(t)\right\}\right]}, \\
& \quad i=m+1, \ldots, 2 m .
\end{aligned}
$$

In the next section, we study the transient analysis of these four adaptive mixture algorithms.

\section{Transient analysis}

In this section, we study the mean and the mean-square transient analysis of the adaptive mixture methods. We start with the affinely constrained combination.

\subsection{Affinely constrained mixture}

We first perform the transient analysis of the mixture weights updated with the EGU algorithm. Then, we continue with the transient analysis of the mixture weights updated with the EG algorithm.

\subsubsection{Unconstrained relative entropy}

For the affinely constrained mixture updated with the EGU algorithm, using Taylor Series, we have the multiplicative update as

$$
\begin{aligned}
\lambda_{1}^{(i)}(t+1) & =\lambda_{1}^{(i)}(t) \exp \left\{\mu e(t)\left(\hat{y}_{i}(t)-\hat{y}_{m}(t)\right)\right\} \\
& =\lambda_{1}^{(i)}(t) \sum_{k=0}^{\infty} \frac{\left(\mu e(t)\left(\hat{y}_{i}(t)-\hat{y}_{m}(t)\right)\right)^{k}}{k !}, \\
\lambda_{2}^{(i)}(t+1) & =\lambda_{2}^{(i)}(t) \exp \left\{-\mu e(t)\left(\hat{y}_{i}(t)-\hat{y}_{m}(t)\right)\right\} \\
& =\lambda_{2}^{(i)}(t) \sum_{k=0}^{\infty} \frac{\left(-\mu e(t)\left(\hat{y}_{i}(t)-\hat{y}_{m}(t)\right)\right)^{k}}{k !},
\end{aligned}
$$

for $i=1, \ldots, m-1$. If $e(t)$ and $\hat{y}_{i}(t)-\hat{y}_{m}(t)$ for each $i=1, \ldots$, $m-1$ are bounded, then we can write (5) and (6) as

$$
\begin{aligned}
& \lambda_{1}^{(i)}(t+1) \approx \lambda_{1}^{(i)}(t)\left(1+\mu e(t)\left(\hat{y}_{i}(t)-\hat{y}_{m}(t)\right)+O\left(\mu^{2}\right)\right), \\
& \lambda_{2}^{(i)}(t+1) \approx \lambda_{2}^{(i)}(t)\left(1-\mu e(t)\left(\hat{y}_{i}(t)-\hat{y}_{m}(t)\right)+O\left(\mu^{2}\right)\right),
\end{aligned}
$$

for $i=1, \ldots, m-1$. Since $\mu$ is usually relatively small [2], we approximate (7) and (8) as

$\lambda_{1}^{(i)}(t+1) \approx \lambda_{1}^{(i)}(t)\left(1+\mu e(t)\left(\hat{y}_{i}(t)-\hat{y}_{m}(t)\right)\right)$,

$\lambda_{2}^{(i)}(t+1) \approx \lambda_{2}^{(i)}(t)\left(1-\mu e(t)\left(\hat{y}_{i}(t)-\hat{y}_{m}(t)\right)\right)$.

In our simulations, we illustrate the accuracy of the approximations (9) and (10) under the mixture framework. Using (9) and (10), we can obtain updates on $\lambda_{1}(t)$ and $\lambda_{2}(t)$ as

$\lambda_{1}(t+1)=(I+\mu e(t) \operatorname{diag}(\delta(t))) \lambda_{1}(t)$,

$\lambda_{2}(t+1)=(I-\mu e(t) \operatorname{diag}(\delta(t))) \lambda_{2}(t)$.

Collecting the weights in $\lambda_{a}(t)=\left[\lambda_{1}(t) ; \lambda_{2}(t)\right]$, using the updates (11) and (12), we can write update on $\lambda_{a}(t)$ as

$\lambda_{a}(t+1)=(I+\mu e(t) \operatorname{diag}(\boldsymbol{u}(t))) \lambda_{a}(t)$,

where $\boldsymbol{u}(t)$ is defined as $\boldsymbol{u}(t) \triangleq[\delta(t) ;-\delta(t)]$.

For the desired signal $y(t)$, we can write $y(t)-\hat{y}_{m}(t)=$ $\lambda_{0}^{T}(t) \delta(t)+e_{0}(t)$, where $\lambda_{0}(t)$ is the optimum MSE solution at time $t$ such that $\lambda_{0}(t) \triangleq \boldsymbol{R}^{-1}(t) \boldsymbol{p}(t), \boldsymbol{R}(t) \triangleq E\left[\boldsymbol{\delta}(t) \boldsymbol{\delta}^{T}(t)\right], \boldsymbol{p}(t) \triangleq$ $E\left\{\boldsymbol{\delta}(t)\left[y(t)-\hat{y}_{m}(t)\right]\right\}$ and $e_{0}(t)$ is zero mean and uncorrelated with $\delta(t)$. We next show that the mixture weights converge to the optimum solution in the steady state such that $\lim _{t \rightarrow \infty} E[\lambda(t)]=$ $\lim _{t \rightarrow \infty} \lambda_{0}(t)$ for properly selected $\mu$.

Subtracting (12) from (11), we obtain

$$
\begin{aligned}
\lambda(t+1)= & \lambda(t)+\mu e(t) \operatorname{diag}(\delta(t))\left(\lambda_{1}(t)+\lambda_{2}(t)\right) \\
= & \lambda(t)-\mu e(t) \operatorname{diag}(\delta(t)) \lambda(t) \\
& +2 \mu e(t) \operatorname{diag}(\delta(t)) \lambda_{1}(t) .
\end{aligned}
$$

Defining $\boldsymbol{\varepsilon}(t) \triangleq \lambda_{0}(t)-\lambda(t)$ and using $e(t)=\delta^{T}(t) \boldsymbol{\varepsilon}(t)+e_{0}(t)$ in (14) yield

$$
\begin{aligned}
\lambda(t+1)= & \lambda(t)-\mu \operatorname{diag}(\delta(t)) \lambda(t) \delta^{T}(t) \boldsymbol{\varepsilon}(t) \\
& -\mu \operatorname{diag}(\delta(t)) \lambda(t) e_{0}(t) \\
& +2 \mu \operatorname{diag}(\delta(t)) \lambda_{1}(t) \delta^{T}(t) \boldsymbol{\varepsilon}(t) \\
& +2 \mu \operatorname{diag}(\delta(t)) \lambda_{1}(t) e_{0}(t) .
\end{aligned}
$$

In (15), subtracting both sides from $\lambda_{0}(t+1)$, we have

$$
\begin{aligned}
\boldsymbol{\varepsilon}(t+1)= & \boldsymbol{\varepsilon}(t)+\mu \operatorname{diag}(\boldsymbol{\delta}(t)) \boldsymbol{\lambda}(t) \boldsymbol{\delta}^{T}(t) \boldsymbol{\varepsilon}(t) \\
& +\mu \operatorname{diag}(\boldsymbol{\delta}(t)) \lambda(t) e_{0}(t) \\
& -2 \mu \operatorname{diag}(\boldsymbol{\delta}(t)) \lambda_{1}(t) \boldsymbol{\delta}^{T}(t) \boldsymbol{\varepsilon}(t) \\
& -2 \mu \operatorname{diag}(\boldsymbol{\delta}(t)) \lambda_{1}(t) e_{0}(t) \\
& +\left[\lambda_{0}(t+1)-\lambda_{0}(t)\right] .
\end{aligned}
$$

Taking expectation of both sides of (16) and using

$E\left[\mu \operatorname{diag}(\delta(t)) \lambda(t) e_{0}(t)\right]=E[\mu \operatorname{diag}(\delta(t)) \lambda(t)] E\left[e_{0}(t)\right]=0$,

$E\left[2 \mu \operatorname{diag}(\delta(t)) \lambda_{1}(t) e_{0}(t)\right]=E\left[2 \mu \operatorname{diag}(\delta(t)) \lambda_{1}(t)\right] E\left[e_{0}(t)\right]=0$,

and assuming that the correlation between $\boldsymbol{\varepsilon}(t)$ and $\lambda_{1}(t), \lambda_{2}(t)$ is small enough to be safely omitted [17] yields

$$
\begin{aligned}
E[\boldsymbol{\varepsilon}(t+1)]= & E\left[I-\mu \operatorname{diag}\left(\lambda_{1}(t)+\lambda_{2}(t)\right) \boldsymbol{\delta}(t) \boldsymbol{\delta}^{T}(t)\right] E[\boldsymbol{\varepsilon}(t)] \\
& +E\left[\lambda_{0}(t+1)-\lambda_{0}(t)\right] .
\end{aligned}
$$


Table 1

Time evolution of the mean and the variance of the affinely constrained mixture weights updated with the EGU algorithm.

$\boldsymbol{q}_{a}(t+1)=\boldsymbol{q}_{a}(t)+\mu \operatorname{diag}(\boldsymbol{\gamma}(t)) \boldsymbol{q}_{a}(t)-\mu \operatorname{diag}\left(\boldsymbol{Q}_{a}(t) \boldsymbol{\Gamma}(t)\right)$,

$\boldsymbol{Q}_{a}(t+1)=\left(I+\mu \operatorname{diag}(\boldsymbol{\gamma}(t))-\mu \operatorname{diag}\left(\boldsymbol{\Gamma}(t) \boldsymbol{q}_{a}(t)\right)\right) \boldsymbol{Q}_{a}(t)-\mu E\left[\operatorname{diag}^{2}(\boldsymbol{u}(t))\right]\left(\boldsymbol{Q}_{a}(t)-\boldsymbol{q}_{a}(t) \boldsymbol{q}_{a}^{T}(t)\right) \mathbf{1} \boldsymbol{q}_{a}^{T}(t)-\mu \operatorname{diag}\left(\boldsymbol{q}_{a}(t)\right) \boldsymbol{\Gamma}(t)\left(\boldsymbol{Q}_{a}(t)-\boldsymbol{q}_{a}(t) \boldsymbol{q}_{a}^{T}(t)\right)$ $+\boldsymbol{Q}_{a}(t)\left(\mu \operatorname{diag}(\boldsymbol{\gamma}(t))-\mu \operatorname{diag}\left(\boldsymbol{\Gamma}(t) \boldsymbol{q}_{a}(t)\right)\right)-\mu \boldsymbol{q}_{a}(t) \mathbf{1}^{T}\left(\boldsymbol{Q}_{a}(t)-\boldsymbol{q}_{a}(t) \boldsymbol{q}_{a}^{T}(t)\right) E\left[\operatorname{diag}^{2}(\boldsymbol{u}(t))\right]-\mu\left(\boldsymbol{Q}_{a}(t)-\boldsymbol{q}_{a}(t) \boldsymbol{q}_{a}^{T}(t)\right) \boldsymbol{\Gamma}(t) \operatorname{diag}\left(\boldsymbol{q}_{a}(t)\right)$.

Assuming convergence of $\boldsymbol{R}(t)$ and $\boldsymbol{p}(t)$ (which is true for a wide range of adaptive methods in the first stage [16,14,21]), we obtain $\lim _{t \rightarrow \infty} E\left[\lambda_{0}(t+1)-\lambda_{0}(t)\right]=0$. If $\mu$ is chosen such that the eigenvalues of $E\left[I-\mu \operatorname{diag}\left(\lambda_{1}(t)+\lambda_{2}(t)\right) \delta(t) \delta^{T}(t)\right]$ have strictly less than unit magnitude for every $t$, then $\lim _{t \rightarrow \infty} E[\lambda(t)]=\lim _{t \rightarrow \infty} \lambda_{0}(t)$.

For the transient analysis of the MSE, we have

$$
\begin{aligned}
E\left[e^{2}(t)\right]= & E\left\{\left[y(t)-\hat{y}_{m}(t)\right]^{2}\right\} \\
& -2 \bar{\lambda}_{a}^{T}(t) E\left\{\left[y(t)-\hat{y}_{m}(t)\right][\delta(t) ;-\delta(t)]\right\} \\
& +E\left\{\lambda_{a}^{T}(t)[\delta(t) ;-\delta(t)][\delta(t) ;-\delta(t)]^{T} \lambda_{a}(t)\right\} \\
= & E\left\{\left[y(t)-\hat{y}_{m}(t)\right]^{2}\right\}-2 \bar{\lambda}_{a}^{T}(t) E\left\{\left[y(t)-\hat{y}_{m}(t)\right] \boldsymbol{u}(t)\right\} \\
& +\operatorname{tr}\left(E\left[\lambda_{a}(t) \lambda_{a}^{T}(t)\right] E\left\{\boldsymbol{u}(t) \boldsymbol{u}(t)^{T}\right\}\right) \\
= & E\left\{\left[y(t)-\hat{y}_{m}(t)\right]^{2}\right\}-2 \bar{\lambda}_{a}^{T}(t) \boldsymbol{\gamma}(t) \\
& +\operatorname{tr}\left(E\left[\lambda_{a}(t) \lambda_{a}^{T}(t)\right] \boldsymbol{\Gamma}(t)\right),
\end{aligned}
$$

where we define $\boldsymbol{\gamma}(t) \triangleq E\left\{\boldsymbol{u}(t)\left[y(t)-\hat{y}_{m}(t)\right]\right\}$ and $\boldsymbol{\Gamma}(t) \triangleq E[\boldsymbol{u}(t) \times$ $\left.\boldsymbol{u}^{T}(t)\right]$

For the recursion of $\bar{\lambda}_{a}(t)=E\left[\lambda_{a}(t)\right]$, using (13), we get

$$
\begin{aligned}
\bar{\lambda}_{a}(t+1)= & \bar{\lambda}_{a}(t)+\mu \operatorname{diag}(\boldsymbol{\gamma}(t)) \bar{\lambda}_{a}(t) \\
& -\mu \operatorname{diag}\left(E\left[\lambda_{a}(t) \lambda_{a}^{T}(t)\right] \Gamma(t)\right) .
\end{aligned}
$$

Using (13) and $e(t)=\left[y(t)-\hat{y}_{m}(t)\right]-\lambda^{T}(t) \delta(t)$, assuming $\lambda_{a}(t)$ is Gaussian and assuming $\lambda_{a}^{(i)}(t)$ and $\lambda_{a}^{(j)}(t)$ are uncorrelated when $i \neq j$ (as in Chapter 9.4.2 of [14], and [17]), defining the diagonal matrix $\boldsymbol{D}(t)=E\left[\lambda_{a}(t) \lambda_{a}^{T}(t)\right]-\bar{\lambda}_{a}(t) \bar{\lambda}_{a}^{T}(t)$ and since $\mu$ is small, ignoring the terms that are proportional to $\mu^{2}$, we get a recursion for $E\left[\lambda_{a}(t) \lambda_{a}^{T}(t)\right]$ as

$$
\begin{aligned}
& E\left[\boldsymbol{\lambda}_{a}(t+1) \lambda_{a}^{T}(t+1)\right] \\
& =E\left[\boldsymbol{\lambda}_{a}(t) \lambda_{a}^{T}(t)\right]+\mu \operatorname{diag}(\boldsymbol{\gamma}(t)) E\left[\boldsymbol{\lambda}_{a}(t) \lambda_{a}^{T}(t)\right] \\
& \quad-\mu \operatorname{diag}\left(\boldsymbol{\Gamma}(t) \overline{\boldsymbol{\lambda}}_{a}(t)\right) E\left[\boldsymbol{\lambda}_{a}(t) \boldsymbol{\lambda}_{a}^{T}(t)\right] \\
& \quad-\mu E\left[\operatorname{diag}^{2}(\boldsymbol{u}(t))\right] \boldsymbol{D}(t) \mathbf{1}_{a}^{T}(t) \\
& \quad-\mu \operatorname{diag}\left(\bar{\lambda}_{a}(t)\right) \boldsymbol{\Gamma}(t) \boldsymbol{D}(t)+\mu E\left[\lambda_{a}(t) \lambda_{a}^{T}(t)\right] \operatorname{diag}(\boldsymbol{\gamma}(t)) \\
& \quad-\mu E\left[\boldsymbol{\lambda}_{a}(t) \lambda_{a}^{T}(t)\right] \operatorname{diag}\left(\boldsymbol{\Gamma}(t) \bar{\lambda}_{a}(t)\right) \\
& \quad-\mu \overline{\boldsymbol{\lambda}}_{a}(t) \mathbf{1}^{T} \boldsymbol{D}(t) E\left[\operatorname{diag}^{2}(\boldsymbol{u}(t))\right] \\
& \quad-\mu \boldsymbol{D}(t) \boldsymbol{\Gamma}(t) \operatorname{diag}\left(\bar{\lambda}_{a}(t)\right) .
\end{aligned}
$$

Defining $\boldsymbol{q}_{a}(t) \triangleq \bar{\lambda}_{a}(t)$ and $\boldsymbol{Q}_{a}(t) \triangleq E\left[\lambda_{a}(t) \lambda_{a}^{T}(t)\right]$, we express (19) and (20) as a coupled recursions in Table 1 .

In Table 1 , we provide the mean and the variance recursions for $\boldsymbol{Q}_{a}(t)$ and $\boldsymbol{q}_{a}(t)$. To implement these recursions, one needs to only provide $\boldsymbol{\Gamma}(t)$ and $\boldsymbol{\gamma}(t)$. Note that $\boldsymbol{\Gamma}(t)$ and $\boldsymbol{\gamma}(t)$ are derived for a wide range of adaptive filters $[16,14]$. If we use the mean and the variance recursions in (18), then we obtain the time evolution of the final MSE. This completes the transient analysis of the affinely constrained mixture weights updated with the EGU algorithm.

\subsubsection{Relative entropy}

For the affinely constrained combination updated with the EG algorithm, we have the multiplicative updates as

$$
\begin{aligned}
\lambda_{1}^{(i)}(t+1)= & u\left(\lambda_{1}^{(i)}(t) \exp \left\{\mu e(t)\left(\hat{y}_{i}(t)-\hat{y}_{m}(t)\right)\right\}\right) \\
& \times\left(\sum _ { k = 1 } ^ { m - 1 } \left[\lambda_{1}^{(k)}(t) \exp \left\{\mu e(t)\left(\hat{y}_{k}(t)-\hat{y}_{m}(t)\right)\right\}\right.\right. \\
& \left.\left.+\lambda_{2}^{(k)}(t) \exp \left\{-\mu e(t)\left(\hat{y}_{k}(t)-\hat{y}_{m}(t)\right)\right\}\right]\right)^{-1}, \\
\lambda_{2}^{(i)}(t+1)= & u\left(\lambda_{2}^{(i)}(t) \exp \left\{-\mu e(t)\left(\hat{y}_{i}(t)-\hat{y}_{m}(t)\right)\right\}\right) \\
& \times\left(\sum _ { k = 1 } ^ { m - 1 } \left[\lambda_{1}^{(k)}(t) \exp \left\{\mu e(t)\left(\hat{y}_{k}(t)-\hat{y}_{m}(t)\right)\right\}\right.\right. \\
& \left.\left.+\lambda_{2}^{(k)}(t) \exp \left\{-\mu e(t)\left(\hat{y}_{k}(t)-\hat{y}_{m}(t)\right)\right\}\right]\right)^{-1},
\end{aligned}
$$

for $i=1, \ldots, m-1$. Using the same approximations as in (7)-(10), we obtain

$$
\begin{aligned}
\lambda_{1}^{(i)}(t+1) \approx & u\left(\lambda_{1}^{(i)}(t)\left(1+\mu e(t)\left(\hat{y}_{i}(t)-\hat{y}_{m}(t)\right)\right)\right) \\
& \times\left(\sum _ { k = 1 } ^ { m - 1 } \left[\lambda_{1}^{(k)}(t)\left(1+\mu e(t)\left(\hat{y}_{k}(t)-\hat{y}_{m}(t)\right)\right)\right.\right. \\
& \left.\left.+\lambda_{2}^{(k)}(t)\left(1-\mu e(t)\left(\hat{y}_{k}(t)-\hat{y}_{m}(t)\right)\right)\right]\right)^{-1}, \\
\lambda_{2}^{(i)}(t+1) \approx & u\left(\lambda_{2}^{(i)}(t)\left(1-\mu e(t)\left(\hat{y}_{i}(t)-\hat{y}_{m}(t)\right)\right)\right) \\
& \times\left(\sum _ { k = 1 } ^ { m - 1 } \left[\lambda_{1}^{(k)}(t)\left(1+\mu e(t)\left(\hat{y}_{k}(t)-\hat{y}_{m}(t)\right)\right)\right.\right. \\
& \left.\left.+\lambda_{2}^{(k)}(t)\left(1-\mu e(t)\left(\hat{y}_{k}(t)-\hat{y}_{m}(t)\right)\right)\right]\right)^{-1} .
\end{aligned}
$$

In our simulations, we illustrate the accuracy of the approximations (21) and (22) under the mixture framework. Using (21) and (22), we obtain updates on $\lambda_{1}(t)$ and $\lambda_{2}(t)$ as

$$
\begin{aligned}
& \lambda_{1}(t+1)=u \frac{(I+\mu e(t) \operatorname{diag}(\delta(t))) \lambda_{1}(t)}{\left[\mathbf{1}^{T}+\mu e(t) \boldsymbol{u}^{T}(t)\right] \lambda_{a}(t)}, \\
& \lambda_{2}(t+1)=u \frac{(I-\mu e(t) \operatorname{diag}(\delta(t))) \lambda_{2}(t)}{\left[\mathbf{1}^{T}+\mu e(t) \boldsymbol{u}^{T}(t)\right] \lambda_{a}(t)} .
\end{aligned}
$$

Using updates (23) and (24), we can write update on $\lambda_{a}(t)$

$\lambda_{a}(t+1)=u \frac{[I+\mu e(t) \operatorname{diag}(\boldsymbol{u}(t))] \lambda_{a}(t)}{\left[\mathbf{1}^{T}+\mu e(t) \boldsymbol{u}^{T}(t)\right] \lambda_{a}(t)}$.

For the recursion of $\bar{\lambda}_{a}(t)$, using (25), we get

$$
\begin{aligned}
E & {\left[\lambda_{a}(t+1)\right] } \\
& =E\left\{u \frac{[I+\mu e(t) \operatorname{diag}(\boldsymbol{u}(t))] \lambda_{a}(t)}{\left[\mathbf{1}^{T}+\mu e(t) \boldsymbol{u}^{T}(t)\right] \lambda_{a}(t)}\right\}
\end{aligned}
$$




$$
\begin{aligned}
& \approx u \frac{E\left\{[I+\mu e(t) \operatorname{diag}(\boldsymbol{u}(t))] \lambda_{a}(t)\right\}}{E\left\{\left[\mathbf{1}^{T}+\mu e(t) \boldsymbol{u}^{T}(t)\right] \lambda_{a}(t)\right\}} \\
& =u \frac{E\left[\lambda_{a}(t)\right]+\mu \operatorname{diag}(\boldsymbol{\gamma}(t)) E\left[\lambda_{a}(t)\right]-\mu \operatorname{diag}\left(E\left[\lambda_{a}(t) \lambda_{a}^{T}(t)\right] \boldsymbol{\Gamma}(t)\right)}{\left[\mathbf{1}^{T}+\mu \boldsymbol{\gamma}^{T}(t)\right] E\left[\lambda_{a}(t)\right]-\mu \operatorname{tr}\left(E\left[\lambda_{a}(t) \lambda_{a}^{T}(t)\right] \boldsymbol{\Gamma}(t)\right)},
\end{aligned}
$$

where in (26) we approximate expectation of the quotient with the quotient of the expectations. In our simulations, we also illustrate the accuracy of this approximation in the mixture framework. From (25), using the same approximation in (27), assuming $\lambda_{a}(t)$ is Gaussian, assuming $\lambda_{a}^{(i)}(t)$ and $\lambda_{a}^{(j)}(t)$ are uncorrelated when $i \neq j$, and since $\mu$ is small, ignoring the terms that are proportional to $\mu^{2}$, we get a recursion for $E\left[\lambda_{a}(t) \lambda_{a}^{T}(t)\right]$ as

$E\left[\lambda_{a}(t+1) \lambda_{a}^{T}(t+1)\right]=u^{2} \frac{\boldsymbol{A}(t)}{b(t)}$,

where $\boldsymbol{A}(t)$ is equal to the right-hand side of (20) and

$$
\begin{aligned}
b(t)= & \mathbf{1}^{T} E\left[\lambda_{a}(t) \lambda_{a}^{T}(t)\right] \mathbf{1}+\mu \boldsymbol{p}^{T}(t) E\left[\lambda_{a}(t) \lambda_{a}^{T}(t)\right] \mathbf{1} \\
& -\mu \bar{\lambda}_{a}^{T}(t) \boldsymbol{R}(t) E\left[\lambda_{a}(t) \lambda_{a}^{T}(t)\right] \mathbf{1}-\mu \mathbf{1}^{T} \boldsymbol{D}(t) \boldsymbol{R}(t) \bar{\lambda}_{a}(t) \\
& -\mu \mathbf{1}^{T} \boldsymbol{D}(t) E\left[\operatorname{diag}^{2}(\boldsymbol{u}(t))\right] \mathbf{1}^{T} \bar{\lambda}_{a}(t) \mathbf{1} \\
& +\mu \mathbf{1}^{T} E\left[\lambda_{a}(t) \lambda_{a}^{T}(t)\right] \boldsymbol{p}(t)-\mu \mathbf{1}^{T} E\left[\lambda_{a}(t) \lambda_{a}^{T}(t)\right] \boldsymbol{R}(t) \bar{\lambda}_{a}(t) \\
& -\mu \bar{\lambda}_{a}^{T}(t) \boldsymbol{R}(t) \boldsymbol{D}(t) \mathbf{1}-\mu \mathbf{1}^{T} \bar{\lambda}_{a}^{T}(t) \mathbf{1} E\left[\operatorname{diag}^{2}(\boldsymbol{u}(t))\right] \boldsymbol{D}(t) .
\end{aligned}
$$

If we use the mean (27) and the variance (28), (29) recursions in (18), then we obtain the time evolution of the final MSE. This completes the transient analysis of the affinely constrained mixture weights updated with the EG algorithm.

\subsection{Unconstrained mixture}

We use the unconstrained relative entropy and the relative entropy as distance measures to update unconstrained mixture weights. We first perform transient analysis of the mixture weights updated using the EGU algorithm. Then, we continue with the transient analysis of the mixture weights updated using the EG algorithm. Note that since the unconstrained case is close to the affinely constrained case, we only provide the necessary modifications to get the mean and the variance recursions for the transient analysis.

\subsubsection{Unconstrained relative entropy}

For the unconstrained combination updated with EGU, we have the multiplicative updates as

$w_{1}^{(i)}(t+1)=w_{1}^{(i)}(t) \exp \left\{\mu e(t) \hat{y}_{i}(t)\right\}$

$w_{2}^{(i)}(t+1)=w_{2}^{(i)}(t) \exp \left\{-\mu e(t) \hat{y}_{i}(t)\right\}$,

for $i=1, \ldots, m$. Using the same approximations as in (7)-(10), we can obtain updates on $\boldsymbol{w}_{1}(t)$ and $\boldsymbol{w}_{2}(t)$ as

$$
\begin{aligned}
& \boldsymbol{w}_{1}(t+1) \approx(I+\mu e(t) \operatorname{diag}(\boldsymbol{x}(t))) \boldsymbol{w}_{1}(t), \\
& \boldsymbol{w}_{2}(t+1) \approx(I-\mu e(t) \operatorname{diag}(\boldsymbol{x}(t))) \boldsymbol{w}_{2}(t) .
\end{aligned}
$$

Collecting the weights in $\boldsymbol{w}_{a}(t)=\left[\boldsymbol{w}_{1}(t) ; \boldsymbol{w}_{2}(t)\right]$, using the updates (30) and (31), we can write update on $\boldsymbol{w}_{a}(t)$ as

$\boldsymbol{w}_{a}(t+1)=(I+\mu e(t) \operatorname{diag}(\boldsymbol{u}(t))) \boldsymbol{w}_{a}(t)$,

where $\boldsymbol{u}(t)$ is defined as $\boldsymbol{u}(t) \triangleq[\boldsymbol{x}(t) ;-\boldsymbol{x}(t)]$.

For the desired signal $y(t)$, we can write $y(t)=\boldsymbol{w}_{0}^{T}(t) \times$ $\boldsymbol{x}(t)+e_{0}(t)$, where $\boldsymbol{w}_{0}(t)$ is the optimum MSE solution at time $t$ such that $\boldsymbol{w}_{0}(t) \triangleq \boldsymbol{R}^{-1}(t) \boldsymbol{p}(t), \quad \boldsymbol{R}(t) \triangleq E\left[\boldsymbol{x}(t) \boldsymbol{x}^{T}(t)\right], \quad \boldsymbol{p}(t) \triangleq$ $E\{\boldsymbol{x}(t) y(t)\}$ and $e_{0}(t)$ is zero-mean disturbance uncorrelated to $\boldsymbol{x}(t)$.
To show that the mixture weights converge to the optimum solution in the steady state such that $\lim _{t \rightarrow \infty} E[\boldsymbol{w}(t)]=\lim _{t \rightarrow \infty} \boldsymbol{w}_{0}(t)$, we follow similar lines as in Section 4.1.1. We modify (14)-(17) such that $\lambda$ will be replaced by $\boldsymbol{w}, \delta(t)$ will be replaced by $\boldsymbol{x}(t)$ and $\boldsymbol{\varepsilon}(t)=\boldsymbol{w}_{0}(t)-\boldsymbol{w}(t)$. After these replacements, we obtain

$$
\begin{aligned}
E[\boldsymbol{\varepsilon}(t+1)]= & E\left[I-\mu \operatorname{diag}\left(\boldsymbol{w}_{1}(t)+\boldsymbol{w}_{2}(t)\right) \boldsymbol{x}(t) \boldsymbol{x}^{T}(t)\right] E[\boldsymbol{\varepsilon}(t)] \\
& +E\left[\boldsymbol{w}_{0}(t+1)-\boldsymbol{w}_{0}(t)\right] .
\end{aligned}
$$

Since, we have $\lim _{t \rightarrow \infty} E\left[\boldsymbol{w}_{0}(t+1)-\boldsymbol{w}_{0}(t)\right]=0$ for most adaptive filters in the first stage [14] and if $\mu$ is chosen so that all the eigenvalues of $E\left[I-\mu \operatorname{diag}\left(\boldsymbol{w}_{1}(t)+\boldsymbol{w}_{2}(t)\right) \boldsymbol{x}(t) \boldsymbol{x}^{T}(t)\right]$ have strictly less than unit magnitude for every $t$, then $\lim _{t \rightarrow \infty} E[\boldsymbol{w}(t)]=$ $\lim _{t \rightarrow \infty} \boldsymbol{w}_{0}(t)$.

For the transient analysis of MSE, defining $\boldsymbol{\gamma}(t) \triangleq E\{\boldsymbol{u}(t) y(t)\}$ and $\boldsymbol{\Gamma}(t) \triangleq E\left[\boldsymbol{u}(t) \boldsymbol{u}^{T}(t)\right]$, (18) is modified as

$$
\begin{aligned}
E\left[e^{2}(t)\right]= & E\left\{y^{2}(t)\right\}-2 \overline{\boldsymbol{w}}_{a}^{T}(t) \boldsymbol{\gamma}(t) \\
& +\operatorname{tr}\left(E\left[\boldsymbol{w}_{a}(t) \boldsymbol{w}_{a}^{T}(t)\right] \boldsymbol{\Gamma}(t)\right) .
\end{aligned}
$$

Accordingly, we modify the mean recursion (19) and the variance recursion (20) such that instead of $\lambda_{a}(t)$ we use $\boldsymbol{w}_{a}(t)$. We also modify Table 1 using $\boldsymbol{q}_{a}(t) \triangleq \overline{\boldsymbol{w}}_{a}(t)$ and $\boldsymbol{Q}_{a}(t) \triangleq E\left[\boldsymbol{w}_{a}(t) \boldsymbol{w}_{a}^{T}(t)\right]$. If we use this modified mean and variance recursions in (34), then we obtain the time evolution of the final MSE. This completes the transient analysis of the unconstrained mixture weights updated with the EGU algorithm.

\subsubsection{Relative entropy}

For the unconstrained combination updated with the EG algorithm, we have the multiplicative updates as

$$
\begin{aligned}
& w_{a}^{(i)}(t+1) \\
& =u \frac{w_{a}^{(i)}(t) \exp \left\{\mu e(t) \hat{y}_{i}(t)\right\}}{\sum_{k=1}^{m}\left[w_{a}^{(k)}(t) \exp \left\{\mu e(t) \hat{y}_{k}(t)\right\}+w_{a}^{(k+m)}(t) \exp \left\{-\mu e(t) \hat{y}_{k}(t)\right\}\right]}, \\
& \quad i=1, \ldots, m, \\
& w_{a}^{(i)}(t+1) \\
& =u \frac{w_{a}^{(i)}(t) \exp \left\{-\mu e(t) \hat{y}_{i}(t)\right\}}{\sum_{k=1}^{m}\left[w_{a}^{(k)}(t) \exp \left\{\mu e(t) \hat{y}_{k}(t)\right\}+w_{a}^{(k+m)}(t) \exp \left\{-\mu e(t) \hat{y}_{k}(t)\right\}\right]}, \\
& i=m+1, \ldots, 2 m .
\end{aligned}
$$

Following similar lines, we modify (23)-(25), (27)-(29) such that we replace $\delta(t)$ with $\boldsymbol{x}(t), \lambda$ with $\boldsymbol{w}$ and $\boldsymbol{u}(t)=[\boldsymbol{x}(t) ;-\boldsymbol{x}(t)]$. Finally, we use the modified mean and variance recursions in (34) and obtain the time evolution of the final MSE. This completes the transient analysis of the unconstrained mixture weights updated with the EG algorithm.

\section{Simulations}

In this section, we illustrate the accuracy of our results and compare performances of different adaptive mixture methods through simulations. In our simulations, we observe that using the EG algorithm to train the mixture weights yields better performance compared to using the LMS algorithm or the EGU algorithm to train the mixture weights for combinations having more than two filters and when the combination favors only a few of the constituent filters. The LMS algorithm and the EGU algorithm perform similarly in our simulations when they are used to train the mixture weights. We also observe in our simulations that the mixture weights under the EG update converge to the optimum combination vector faster than the mixture weights under the LMS algorithm. 


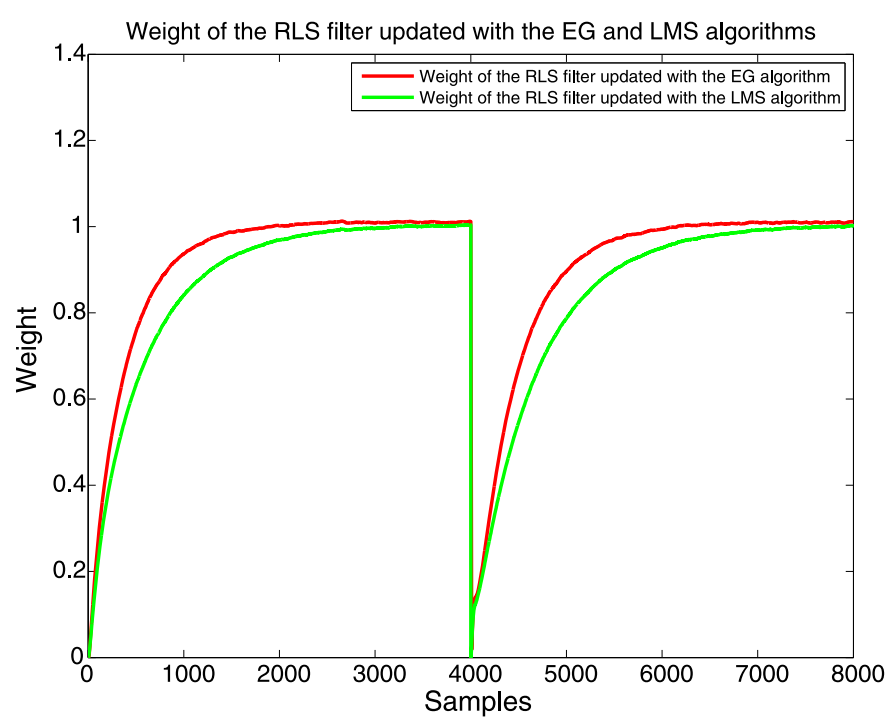

(a)

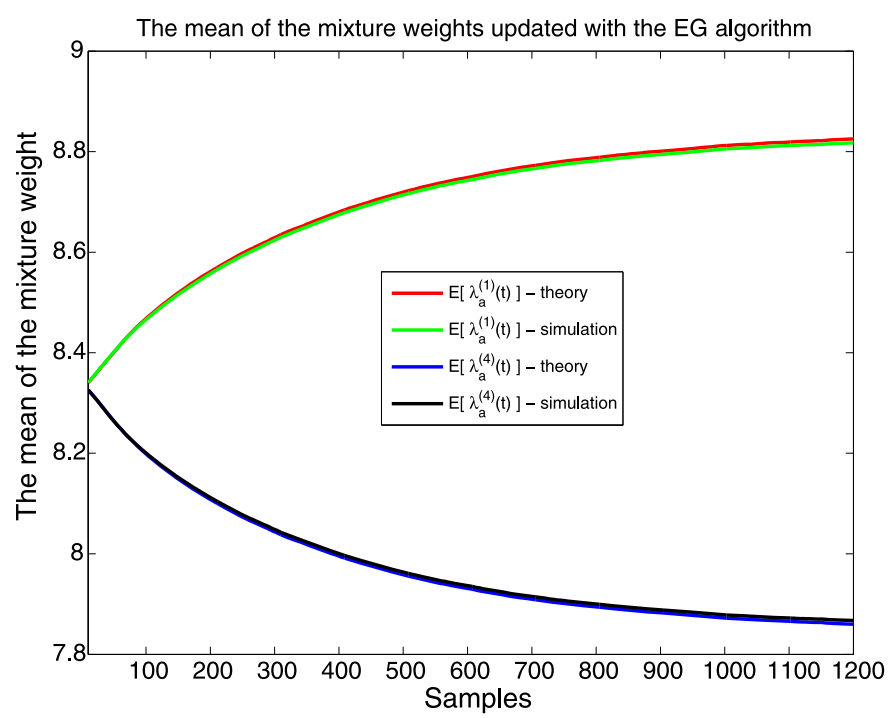

(c)

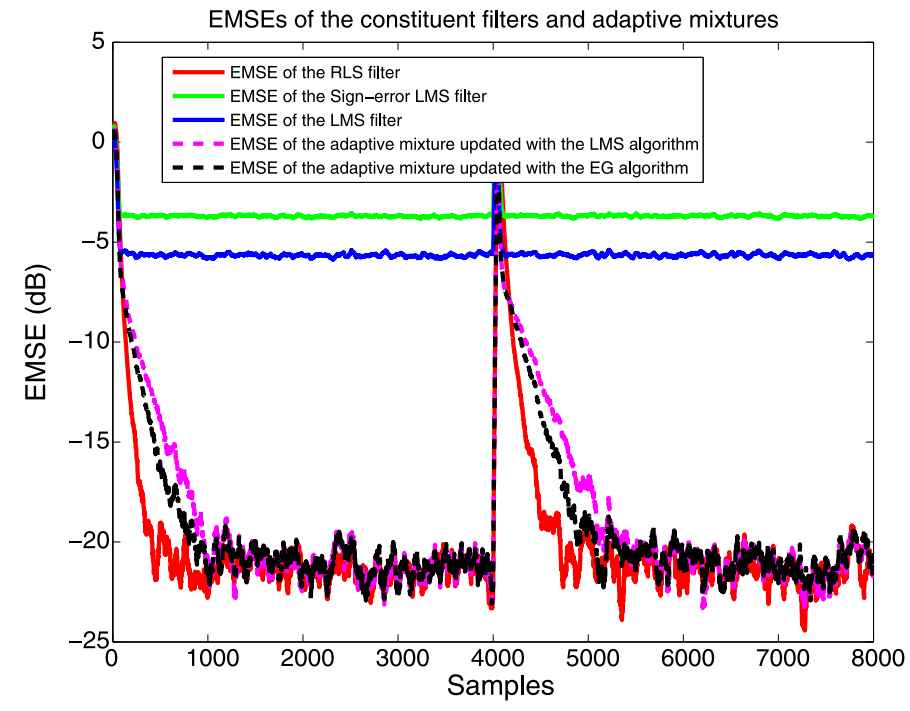

(b)

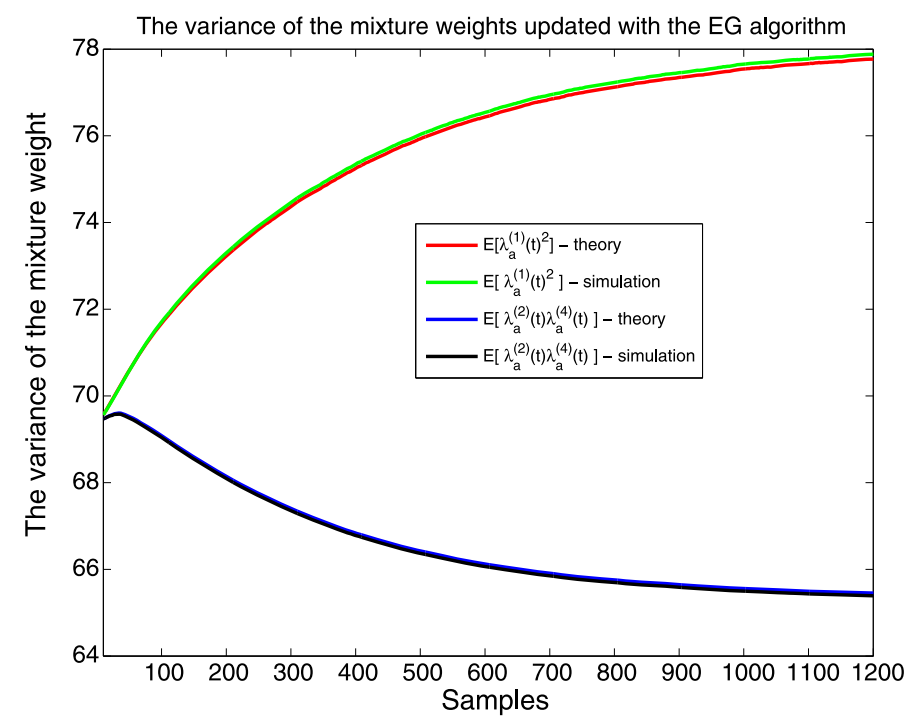

(d)

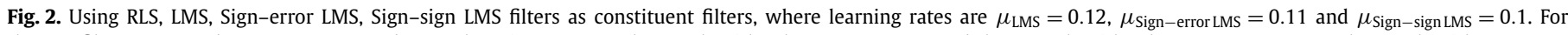

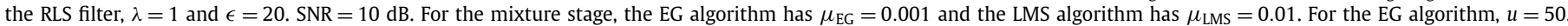

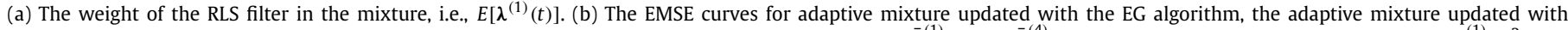

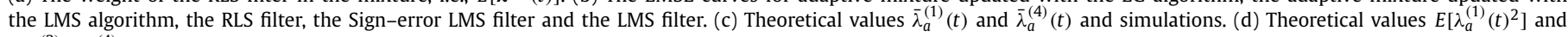
$E\left[\lambda_{a}^{(2)}(t) \lambda_{a}^{(4)}(t)\right]$ and simulations.

To compare performances of the EG and LMS algorithms and illustrate the accuracy of our results in (27)-(29) under different algorithmic parameters, the desired signal as well as the system parameters are selected as follows. First, a seventh-order linear filter, $\boldsymbol{w}_{0}=[0.25,-0.47,-0.37,0.045,-0.18,0.78,0.147]^{T}$, is chosen as in [17]. The underlying signal is generated using the data model $y(t)=\tau \boldsymbol{w}_{0}^{T} \boldsymbol{a}(t)+n(t)$, where $\boldsymbol{a}(t)$ is an i.i.d. Gaussian vector process with zero-mean and unit variance entries, i.e., $E\left[\boldsymbol{a}(t) \boldsymbol{a}^{T}(t)\right]=\boldsymbol{I}, n(t)$ is an i.i.d. Gaussian noise process with zeromean and variance $E\left[n^{2}(t)\right]=0.3$, and $\tau$ is a positive scalar to control SNR. Hence, the SNR of the desired signal is given by $\mathrm{SNR} \triangleq 10 \log \left(\frac{E\left[\tau^{2}\left(\boldsymbol{w}_{0}^{T} \boldsymbol{u}(t)\right)^{2}\right]}{0.01}\right)=10 \log \left(\frac{\tau^{2}\left\|\boldsymbol{w}_{o}\right\|^{2}}{0.01}\right)$.

For the first experiment, we have SNR $=10 \mathrm{~dB}$. To model the unknown system we use four linear filters using the RLS algorithm, LMS algorithm, Sign-error LMS algorithm and Sign-sign LMS algorithm. We emphasize that depending on the underlying signal and/or application, one of these algorithms is prefer- able to the others, however, such a selection is only possible in hindsight. Hence, an adaptive combination could resolve such uncertainty [12]. In this experiment, there is a sudden change in the desired signal such that the target $\boldsymbol{w}_{0}$ changes in the middle of the simulations as seen in Fig. 2. In the start of the simulations, the desired signal is generated from a seventh-order linear filter $\boldsymbol{w}_{0}=[0.25,-0.47,-0.37,0.045,-0.18,0.78,0.147]^{T}[17]$, which is then replaced by $\boldsymbol{w}_{0}=[0.62,0.81,-0.74,0.82,0.26,-0.80$, $-0.44]^{T}$ at the 4000th sample. The constituent RLS algorithm is initialized after $\boldsymbol{w}_{0}$ is updated. The learning rates of these constituent filters are set to $\mu_{\mathrm{LMS}}=0.12, \mu_{\text {Sign-error LMS }}=0.11$ and $\mu_{\text {Sign-sign LMS }}=0.1$. The parameters for the RLS algorithm are set to $\lambda=1$ and $\epsilon=20$. Therefore, in the steady state, we obtain the optimum combination vector approximately as $\lambda_{o}=[1,0,0,0]^{T}$, i.e., the final combination vector is sparse. In the second stage, we train the combination weights with the EG and LMS algorithms and compare performances of these algorithms. The EG algorithm 


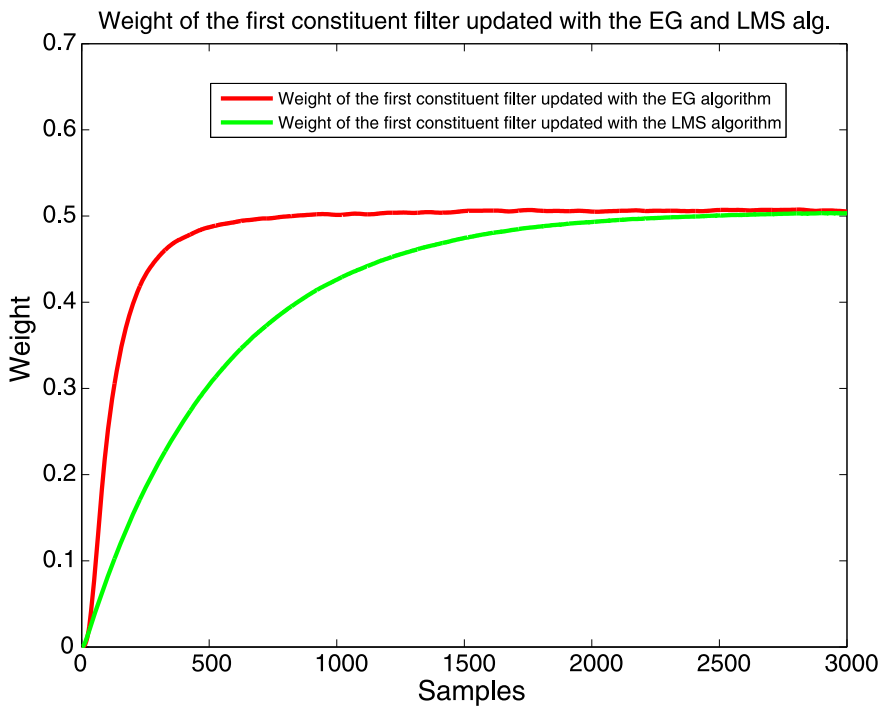

(a)

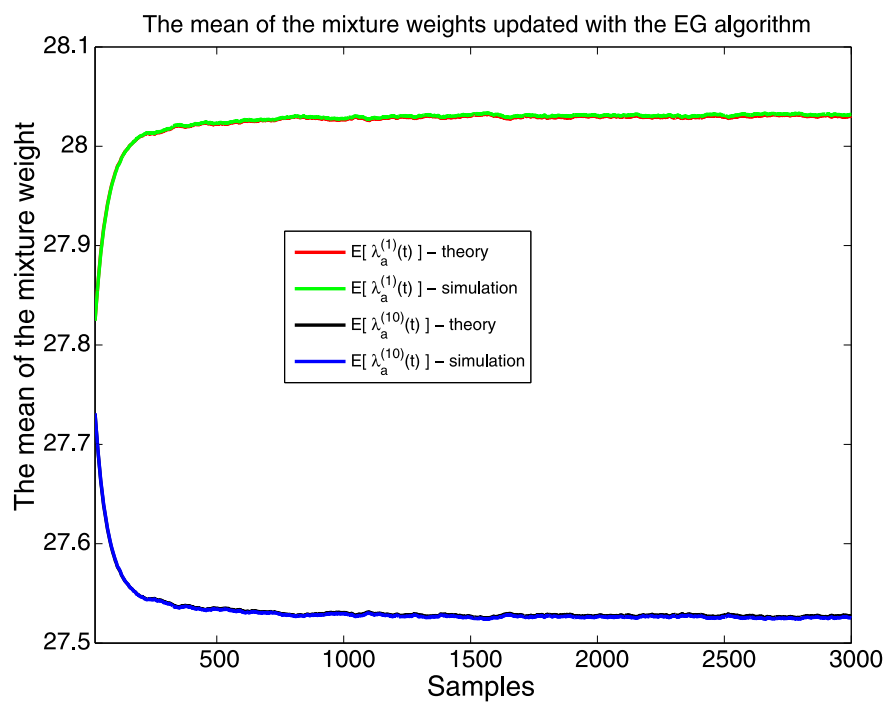

(c)

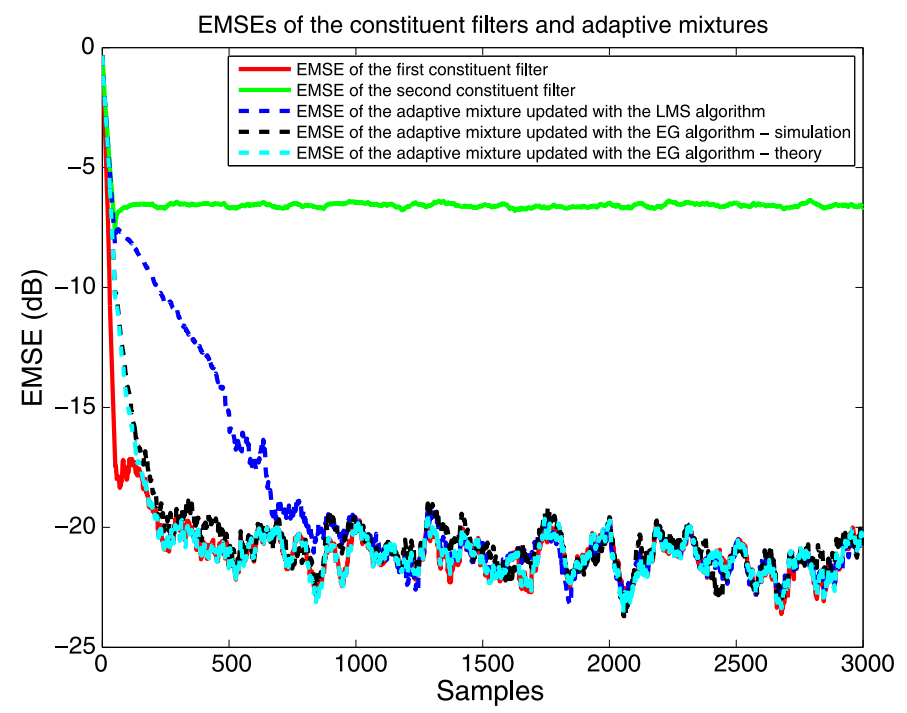

(b)

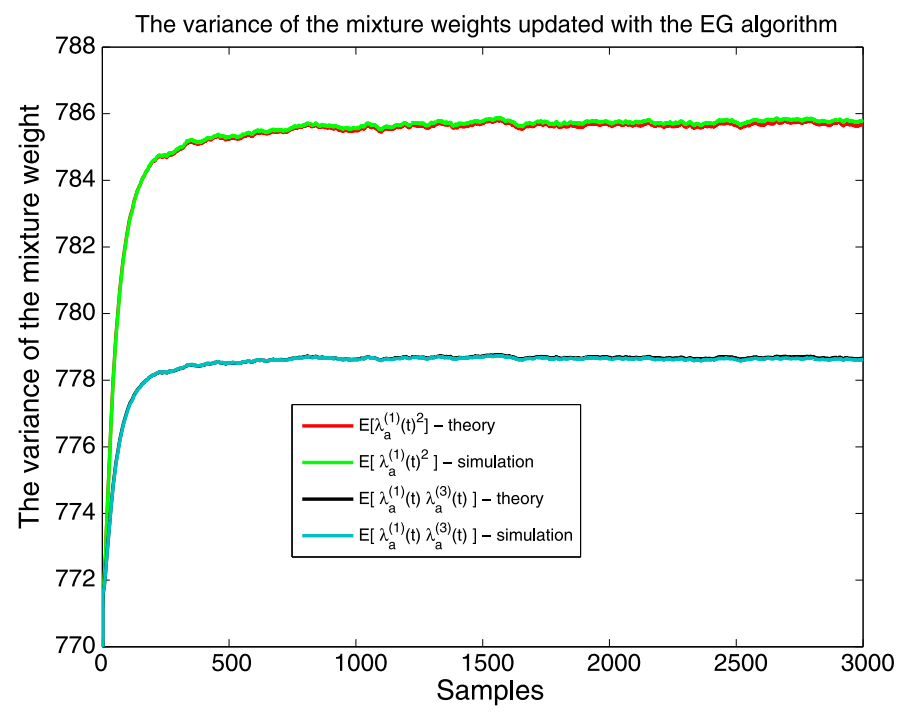

(d)

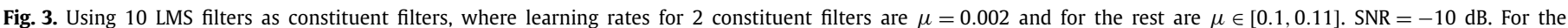

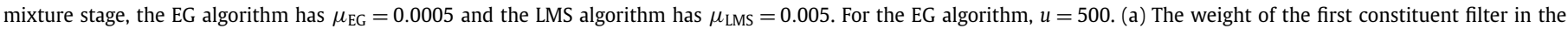

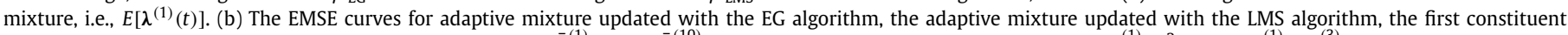
filter and the second constituent filter. (c) Theoretical values $\bar{\lambda}_{a}^{(1)}(t)$ and $\bar{\lambda}_{a}^{(10)}(t)$ and simulations. (d) Theoretical values $E\left[\lambda_{a}^{(1)}(t)^{2}\right]$ and $E\left[\lambda_{a}^{(1)}(t) \lambda_{a}^{(3)}(t)\right]$ and simulations.

has two parameters to adjust while the LMS algorithm has only one parameter to adjust. For the second stage, the learning rates for the EG and LMS algorithms are selected as $\mu_{\mathrm{EG}}=0.001$ and $\mu_{\mathrm{LMS}}=0.01$ such that the EMSEs of both mixtures converge to the same final EMSE to provide a fair comparison. However, there exist a wide range of values for the step sizes so that the algorithms converge to very similar EMSEs. We select $u=50$ for the EG algorithm. In Fig. 2(a), we plot the weight of the RLS filter, i.e. $E\left[\lambda^{(1)}(t)\right]$, updated with the EG and LMS algorithms. In Fig. 2(b), we plot the EMSE curves for the adaptive mixture updated with the EG algorithm, the adaptive mixture updated with the LMS algorithm, the RLS filter with $\lambda=1$ and $\epsilon=20$, the Signerror LMS filter with $\mu_{\text {Sign-error LMS }}=0.11$ and the LMS filter with $\mu_{\mathrm{LMS}}=0.12$. From Figs. 2 (a) and (b), we see that the EG algorithm performs better than the LMS algorithm such that the combination weight under the update of the EG algorithm converges to 1 faster than the combination weight under the update of the LMS algorithm. We also observe from these simulations that even after the sudden change in the statistics, the EG algorithm quickly recovers and performs better than the LMS algorithm. Furthermore the EMSE of the adaptive mixture updated with the EG algorithm converges faster than the EMSE of the adaptive mixture updated with the LMS algorithm. In Fig. 2(c), we plot the theoretical values for $\bar{\lambda}_{a}^{(1)}(t)$ and $\bar{\lambda}_{a}^{(4)}(t)$ along with simulations. Note that in Fig. 2(c) we observe that $\bar{\lambda}^{(1)}(t)=\bar{\lambda}_{a}^{(1)}(t)-\bar{\lambda}_{a}^{(4)}(t)$ converges to 1 as predicted in our derivations. In Fig. 2(d), we plot the theoretical values of $E\left[\lambda_{a}^{(1)}(t)^{2}\right]$ and $E\left[\lambda_{a}^{(2)}(t) \lambda_{a}^{(4)}(t)\right]$ along with simulations. As we observe from Figs. 2 (c) and (d), there is a close agreement between our results and simulations in these experiments. We observe similar results for the other cross terms.

We next model the unknown system using ten linear filters with the LMS update as the constituent filters. For this experiment, we have $\mathrm{SNR}=-10 \mathrm{~dB}$. The learning rates of two constituent filters are set to $\mu_{1}=0.002$ and $\mu_{6}=0.002$ while the learning rates for the rest of the constituent filters are selected randomly in $[0.1,0.11]$. Therefore, in the steady state, we 


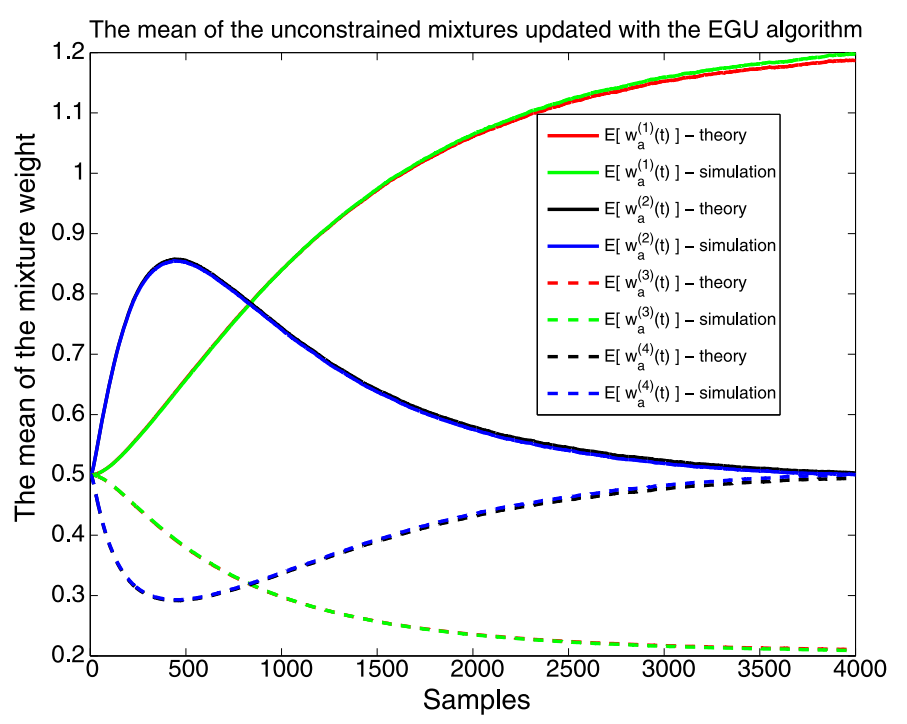

(a)

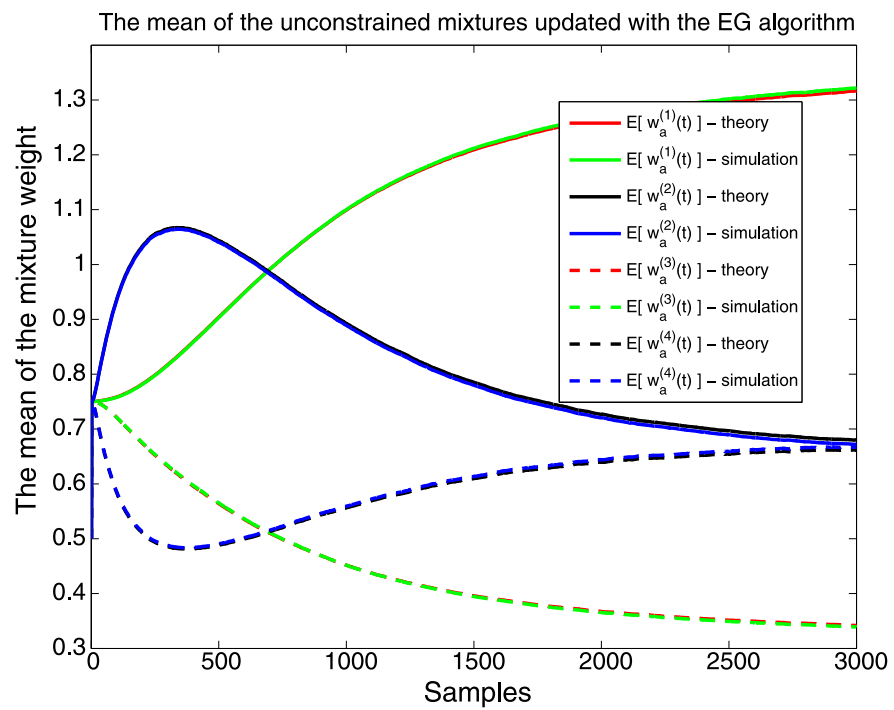

(c)

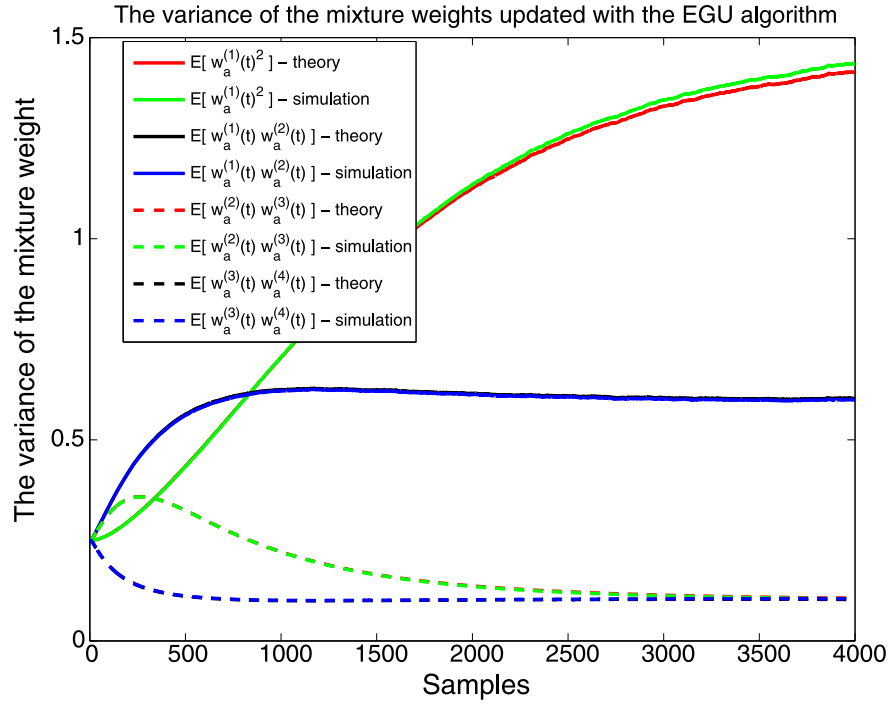

(b)

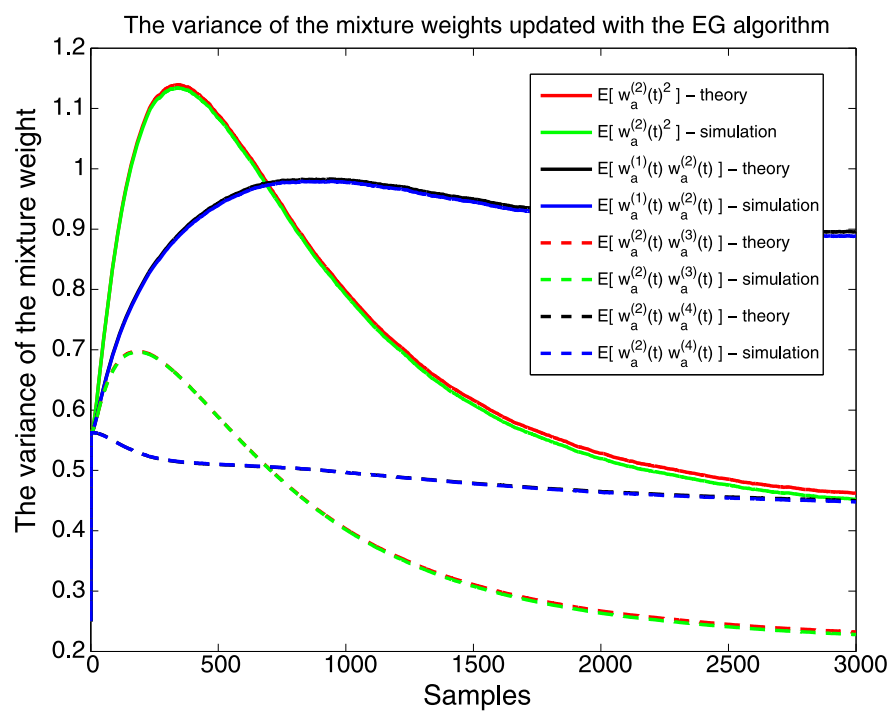

(d)

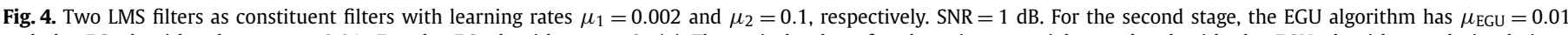

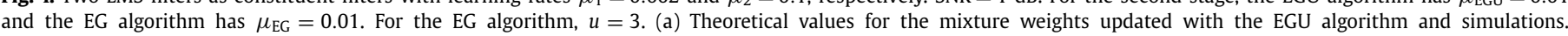

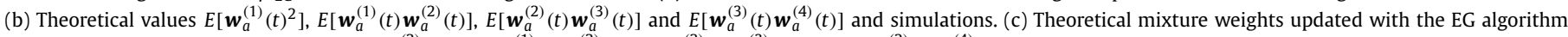
and simulations. (d) Theoretical values $E\left[\boldsymbol{w}_{a}^{(2)}(t)^{2}\right], E\left[\boldsymbol{w}_{a}^{(1)}(t) \boldsymbol{w}_{a}^{(2)}(t)\right], E\left[\boldsymbol{w}_{a}^{(2)}(t) \boldsymbol{w}_{a}^{(3)}(t)\right]$ and $E\left[\boldsymbol{w}_{a}^{(2)}(t) \boldsymbol{w}_{a}^{(4)}(t)\right]$ and simulations.

obtain the optimum combination vector approximately as $\lambda_{0}=$ $[0.5,0,0,0,0,0.5,0,0,0,0]^{T}$, i.e., the final combination vector is sparse. In the second stage, we train the combination weights with the EG and LMS algorithms and compare performances of these algorithms. For the second stage, the learning rates for the EG and LMS algorithms are selected as $\mu_{\mathrm{EG}}=0.0005$ and $\mu_{\mathrm{LMS}}=0.005$ such that the EMSEs of both mixtures converge to the same final EMSE to provide a fair comparison. However, there exist a wide range of values for the step sizes so that the algorithms converge to very similar EMSEs. We select $u=500$ for the EG algorithm. In Fig. 3(a), we plot the weight of the first constituent filter with $\mu_{1}=0.002$, i.e. $E\left[\lambda^{(1)}(t)\right]$, updated with the EG and LMS algorithms. In Fig. 3(b), we plot the EMSE curves for the adaptive mixture updated with the EG algorithm, the adaptive mixture updated with the LMS algorithm, the first constituent filter with $\mu_{1}=0.002$ and the second constituent filter with $\mu_{2} \in[0.1,0.11]$. From Figs. 3 (a) and (b), we see that the EG algorithm performs better than the LMS algorithm such that the combination weight under the update of the EG algorithm converges to 0.5 faster than the combination weight under the update of the LMS algorithm. Furthermore the EMSE of the adaptive mixture updated with the EG algorithm converges faster than the EMSE of the adaptive mixture updated with the LMS algorithm. In Fig. 3(c), to test the accuracy of (27), we plot the theoretical values for $\bar{\lambda}_{a}^{(1)}(t)$ and $\bar{\lambda}_{a}^{(10)}(t)$ along with simulations. Note in Fig. 3(c) we observe that $\bar{\lambda}^{(1)}(t)=\bar{\lambda}_{a}^{(1)}(t)-\bar{\lambda}_{a}^{(10)}(t)$ converges to 0.5 as predicted in our derivations. In Fig. $3(\mathrm{~d})$, to test the accuracy of (28) and (29), as an example, we plot the theoretical values of $E\left[\lambda_{a}^{(1)}(t)^{2}\right]$ and $E\left[\lambda_{a}^{(1)}(t) \lambda_{a}^{(3)}(t)\right]$ along with simulations. As we observe from Figs. 3 (c) and (d), there is a close agreement between our results and simulations in these experiments. We observe similar results for the other cross terms.

We next simulate the unconstrained mixtures updated with the EGU and EG algorithms. Here, we have two linear filters and both using the LMS update to train their weight vectors as the constituent filters. The learning rates for two constituent filters are set to $\mu_{1}=0.002$ and $\mu_{2}=0.1$ respectively. Therefore, 


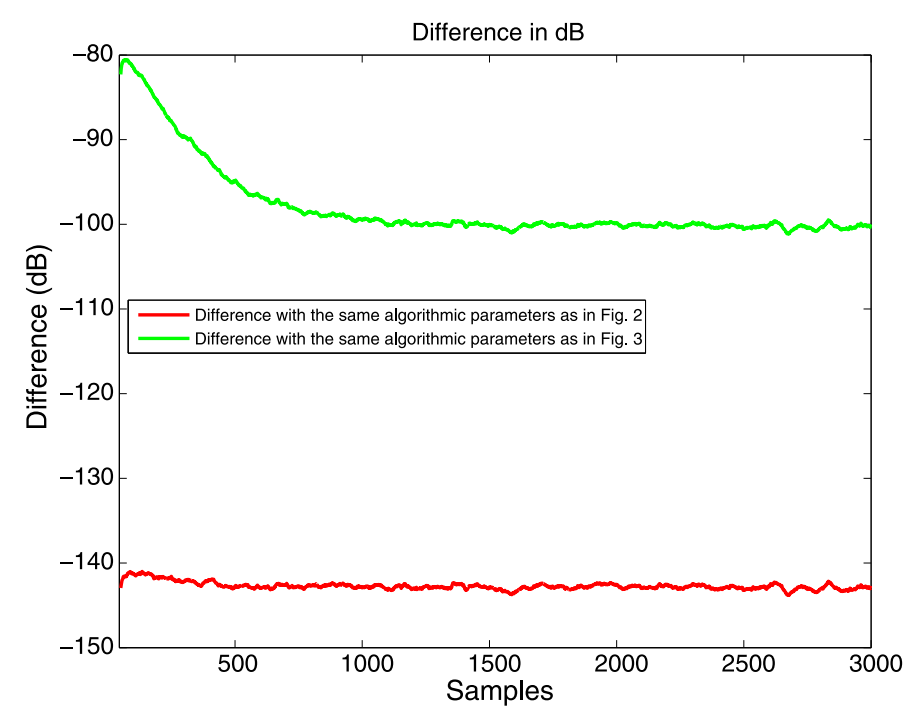

(a)

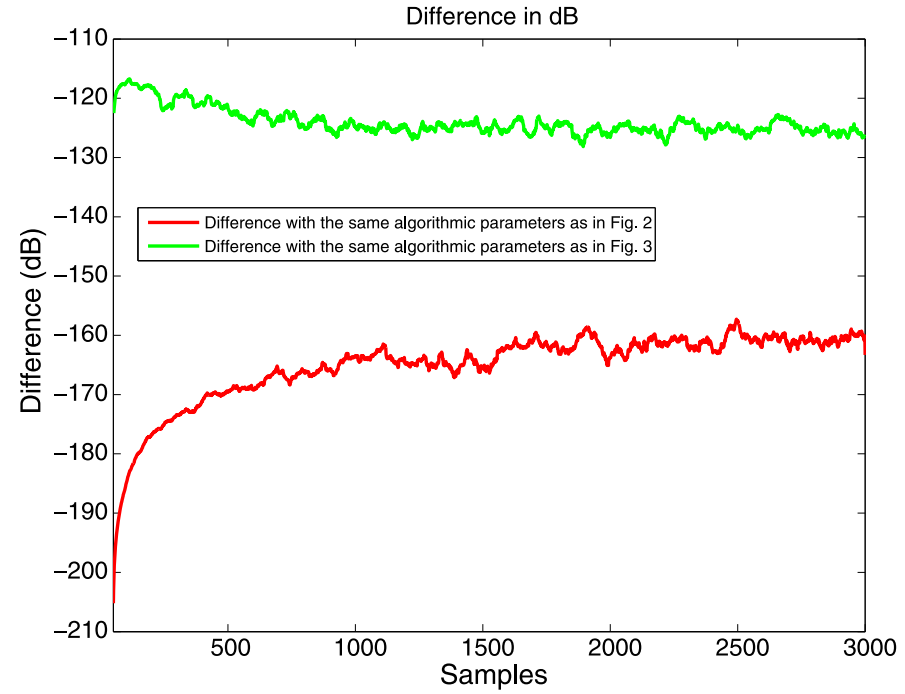

(b)

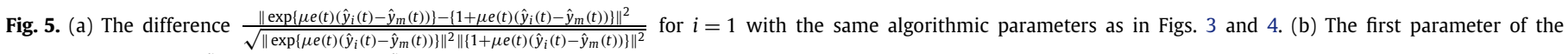
difference $\frac{\left\|E\left\{u \frac{[I+\mu e(t) \operatorname{diag}(\boldsymbol{u}(t))] \lambda_{a}(t)}{\left[\mathbf{1}^{T}+\mu e(t) \boldsymbol{u}^{T}(t)\right] \lambda_{a}(t)}\right\}-u \frac{\left.E[I I+\mu e(t) \operatorname{diag}(\boldsymbol{u}(t))] \lambda_{a}(t)\right\}}{E\left[\left[\mathbf{1}^{T}+\mu e(t) \boldsymbol{u}^{T}(t)\right] \lambda_{a}(t)\right\}}\right\|^{2}}{\sqrt{\left\|E\left\{u \frac{[I+\mu e(t) \operatorname{diag}(\boldsymbol{u}(t))] \lambda_{a}(t)}{\left[\mathbf{1}^{T}+\mu e(t) \boldsymbol{\boldsymbol { T } ^ { T }}(t)\right] \lambda_{a}(t)}\right\}\right\|^{2} \| u \frac{E\left[[I+\mu e(t) \operatorname{diag}(\boldsymbol{u}(t))] \lambda_{a}(t)\right\}}{E\left\{\left[\mathbf{1}^{T}+\mu e(t) \boldsymbol{u}^{T}(t)\right] \lambda_{a}(t)\right\}}}}$ with the same algorithmic parameters as in Figs. 3 and 4.

\begin{tabular}{|l|c|c|c|c|c|c|c|}
\hline & $t=150$ & $t=320$ & $t=560$ & $t=920$ & $t=1210$ & $t=1440$ & $t=1760$ \\
\hline$\lambda_{a}^{(1)}(t)$ & 2.94 & 3.25 & 3.20 & 2.93 & 2.90 & 3.00 & 2.97 \\
\hline$\lambda_{a}^{(2)}(t)$ & 2.71 & 3.05 & 3.43 & 3.02 & 2.96 & 3.13 & 2.88 \\
\hline
\end{tabular}

Fig. 6. Empirical kurtosis values. Experimental setup is from Fig. 4.

in the steady state, we obtain the optimum vector approximately as $\boldsymbol{w}_{0}=[1,0]$. We have SNR $=1$ for these simulations. The unconstrained mixture weights are first updated with the EGU algorithm. For the second stage, the learning rate for the EGU algorithm is selected as $\mu_{\mathrm{EGU}}=0.01$. The theoretical curves in the figures are produced using $\boldsymbol{\Gamma}(t)$ and $\boldsymbol{\gamma}(t)$ that are calculated from the simulations, since our goal is to illustrate the validity of derived equations. In Fig. 4(a), we plot the theoretical values of $\overline{\boldsymbol{w}}_{a}^{(1)}(t)$, $\overline{\boldsymbol{w}}_{a}^{(2)}(t), \overline{\boldsymbol{w}}_{a}^{(3)}(t)$ and $\overline{\boldsymbol{w}}_{a}^{(4)}(t)$ along with simulations. In Fig. 4(b), as an example, we plot the theoretical values of $E\left[\boldsymbol{w}_{a}^{(1)}(t)^{2}\right]$, $E\left[\boldsymbol{w}_{a}^{(1)}(t) \boldsymbol{w}_{a}^{(2)}(t)\right], E\left[\boldsymbol{w}_{a}^{(2)}(t) \boldsymbol{w}_{a}^{(3)}(t)\right]$ and $E\left[\boldsymbol{w}_{a}^{(3)}(t) \boldsymbol{w}_{a}^{(4)}(t)\right]$ along with simulations. We continue to update the mixture weights with the EG algorithm. For the second stage, the learning rate for the EG algorithm is selected as $\mu_{\mathrm{EG}}=0.01$. We select $u=3$ for the EG algorithm. In Fig. $4(\mathrm{c})$, we plot the theoretical values of $\overline{\boldsymbol{w}}_{a}^{(1)}(t)$, $\overline{\boldsymbol{w}}_{a}^{(2)}(t), \overline{\boldsymbol{w}}_{a}^{(3)}(t)$ and $\overline{\boldsymbol{w}}_{a}^{(4)}(t)$ along with simulations. In Fig. $4(\mathrm{~d})$, as an example, we plot the theoretical values of $E\left[\boldsymbol{w}_{a}^{(2)}(t)^{2}\right]$, $E\left[\boldsymbol{w}_{a}^{(1)}(t) \boldsymbol{w}_{a}^{(2)}(t)\right], E\left[\boldsymbol{w}_{a}^{(2)}(t) \boldsymbol{w}_{a}^{(3)}(t)\right]$ and $E\left[\boldsymbol{w}_{a}^{(2)}(t) \boldsymbol{w}_{a}^{(4)}(t)\right]$ along with simulations. We observe a close agreement between our results and simulations.

To test the accuracy of the assumptions in (9) and (10), we plot in Fig. 5(a), the difference

$$
\frac{\left\|\exp \left\{\mu e(t)\left(\hat{y}_{i}(t)-\hat{y}_{m}(t)\right)\right\}-\left\{1+\mu e(t)\left(\hat{y}_{i}(t)-\hat{y}_{m}(t)\right)\right\}\right\|^{2}}{\sqrt{\left\|\exp \left\{\mu e(t)\left(\hat{y}_{i}(t)-\hat{y}_{m}(t)\right)\right\}\right\|^{2}\left\|\left\{1+\mu e(t)\left(\hat{y}_{i}(t)-\hat{y}_{m}(t)\right)\right\}\right\|^{2}}}
$$

for $i=1$ with the same algorithmic parameters as in Figs. 3 and 4. To test the accuracy of the separation assumption in (27), we plot in Fig. 5(b), the first parameter of the difference

$$
\frac{\left\|E\left\{u \frac{[I+\mu e(t) \operatorname{diag}(\boldsymbol{u}(t))] \lambda_{a}(t)}{\left[\mathbf{1}^{T}+\mu e(t) \boldsymbol{u}^{T}(t)\right] \lambda_{a}(t)}\right\}-u \frac{E\left\{[I+\mu e(t) \operatorname{diag}(\boldsymbol{u}(t))] \lambda_{a}(t)\right\}}{E\left\{\left[\mathbf{1}^{T}+\mu e(t) \boldsymbol{u}^{T}(t)\right] \lambda_{a}(t)\right\}}\right\|^{2}}{\sqrt{\left\|E\left\{u \frac{[I+\mu e(t) \operatorname{diag}(\boldsymbol{u}(t))] \lambda_{a}(t)}{\left[\mathbf{1}^{T}+\mu e(t) \boldsymbol{u}^{T}(t)\right] \lambda_{a}(t)}\right\}\right\|^{2}\left\|u \frac{E\left\{[I+\mu e(t) \operatorname{diag}(\boldsymbol{u}(t))] \lambda_{a}(t)\right\}}{E\left\{\left[\mathbf{1}^{T}+\mu e(t) \boldsymbol{u}^{T}(t)\right] \lambda_{a}(t)\right\}}\right\|^{2}}}
$$

with the same algorithmic parameters as in Figs. 3 and 4. We observe that assumptions are fairly accurate for these algorithms in our simulations.

To illustrate the assumption that $\lambda_{a}(t)$ have Gaussian distribution, we calculate the kurtosis of the "empirical" distribution of $\lambda_{a}(t)$ under the setup of the paper. Note that although not rigorous, the kurtosis is often used to measure the closeness of an empirical distribution to a Gaussian distribution [22,23]. For this experiment, we collect 2000 samples of $\lambda_{a}(t)$ under the same algorithmic framework as in Fig. 4 and report the kurtosis values for randomly chosen $t$ 's. The corresponding kurtosis values are provided as a table in Fig. 6. As we observe from Fig. 6, the kurtosis values are close to 3 supporting the assumption that $\lambda_{a}(t)$ follows Gaussian distribution.

To illustrate the assumption of $\lambda_{a}^{(i)}(t)$ and $\lambda_{a}^{(j)}(t)$ are uncorrelated for $j \neq i$, we perform 1000 iterations and plot the ensemble averaged curves that correspond to the difference $\frac{\left\|E\left[\lambda_{a}^{(i)}(t) \lambda_{a}^{(j)}(t)\right]-E\left[\lambda_{a}^{(i)}(t)\right] E\left[\lambda_{a}^{(j)}(t)\right]\right\|}{\sqrt{\sigma^{(i)}}}$ for different randomly chosen $i$ $\sqrt{\left\|E\left[\lambda_{a}^{(i)}(t) \lambda_{a}^{(j)}(t)\right]\right\|^{2}\left\|E\left[\lambda_{a}^{(i)}(t)\right] E\left[\lambda_{a}^{(j)}(t)\right]\right\|^{2}}$

and $j$ parameters with the same algorithmic parameters as in Fig. 3 both for the EG and EGU algorithms. In Fig. 7, we plot this difference for $\lambda_{1}^{(1)}(t)-\lambda_{2}^{(1)}(t)$ and $\lambda_{1}^{(2)}(t)-\lambda_{2}^{(2)}(t)$ pairs. We also plot the difference for $\lambda_{1}^{(3)}(t)-\lambda_{1}^{(8)}(t)$ and $\lambda_{1}^{(4)}(t)-\lambda_{2}^{(6)}(t)$ pairs. As we observe from the plots that it is reasonable to use this assumption to approximate the expectation of the product as the product of the expectations.

In the last simulations, we compare performances of the EGU, EG and LMS algorithms updating the affinely mixture weights under different algorithmic parameters. Algorithmic parameters and constituent filters are selected as in Fig. 3 under SNR $=-5$ and 5 . For the second stage, under $\mathrm{SNR}=-5$, learning rates for the $\mathrm{EG}$, EGU and LMS algorithms are selected as $\mu_{\mathrm{EG}}=0.0005, \mu_{\mathrm{EGU}}=$ 0.005 and $\mu_{\mathrm{LMS}}=0.005$ such that the EMSEs converge to the same final EMSE to provide a fair comparison. However, there exist 


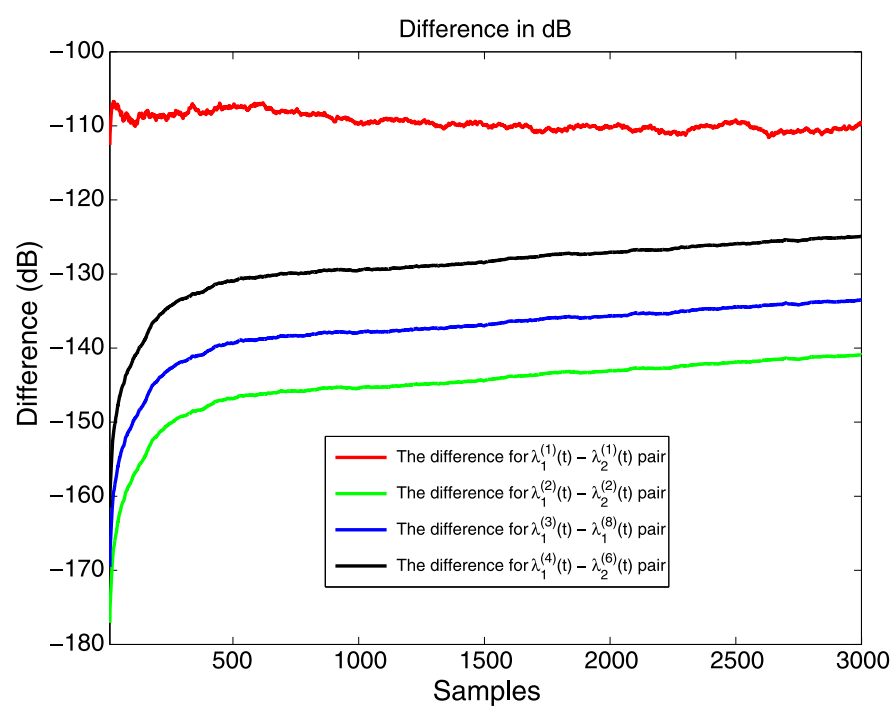

(a)

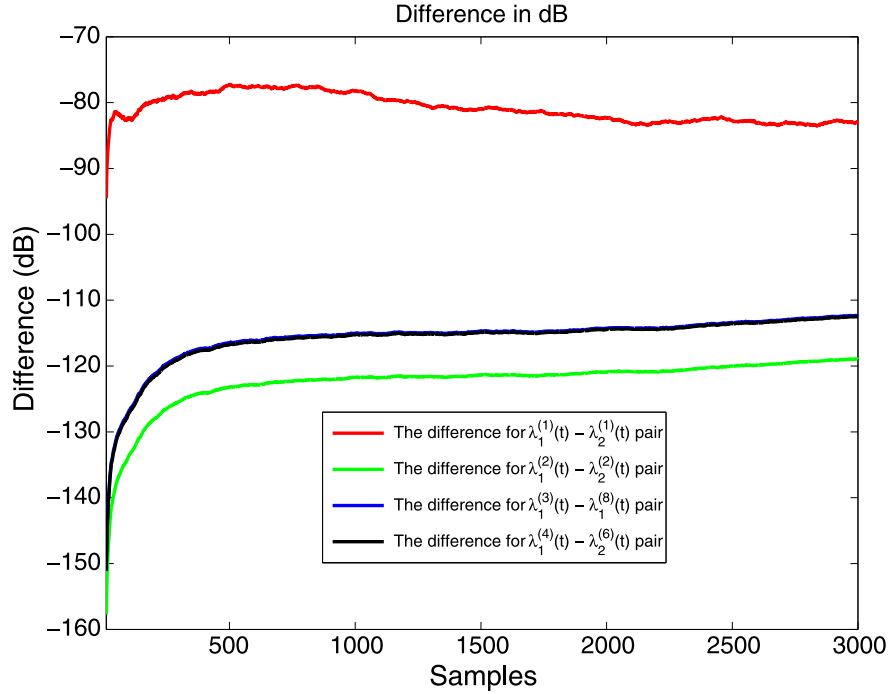

(b)

Fig. 7. (a) The difference $\frac{\left\|E\left[\lambda_{a}^{(i)}(t) \lambda_{a}^{(j)}(t)\right]-E\left[\lambda_{a}^{(i)}(t)\right] E\left[\lambda_{a}^{(j)}(t)\right]\right\|}{\sqrt{\left\|E\left[\lambda_{a}^{(i)}(t) \lambda_{a}^{(j)}(t)\right]\right\|^{2}\left\|E\left[\lambda_{a}^{(i)}(t)\right] E\left[\lambda_{a}^{(j)}(t)\right]\right\|^{2}}}$ for different randomly chosen $i$ and $j$ parameters with the same algorithmic parameters as in Fig. 8(a) for the EG algorithm. (b) The difference $\frac{\left\|E\left[\lambda_{a}^{(i)}(t) \lambda_{a}^{(j)}(t)\right]-E\left[\lambda_{a}^{(i)}(t)\right] E\left[\lambda_{a}^{(j)}(t)\right]\right\|}{\sqrt{\left\|E\left[\lambda_{a}^{(i)}(t) \lambda_{a}^{(j)}(t)\right]\right\|^{2} \| E\left[\lambda_{a}^{(i)}(t)\right] E\left[\lambda_{a}^{(j)}(t) \|^{2}\right.}}$ for different randomly chosen $i$ and $j$ parameters with the same algorithmic parameters as in Fig. $8(\mathrm{a})$ for the EGU algorithm.

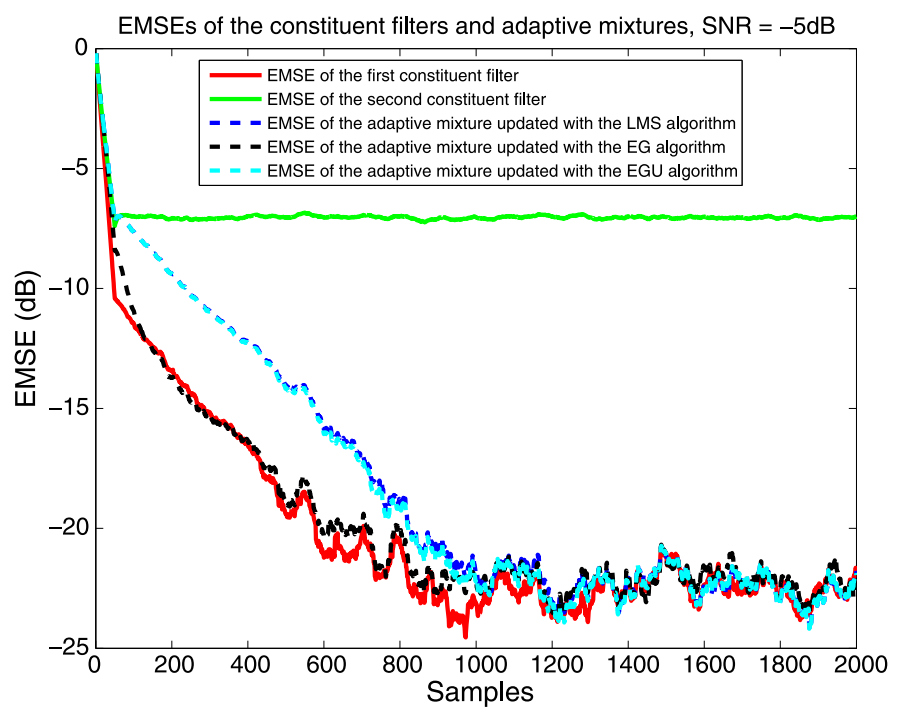

(a)

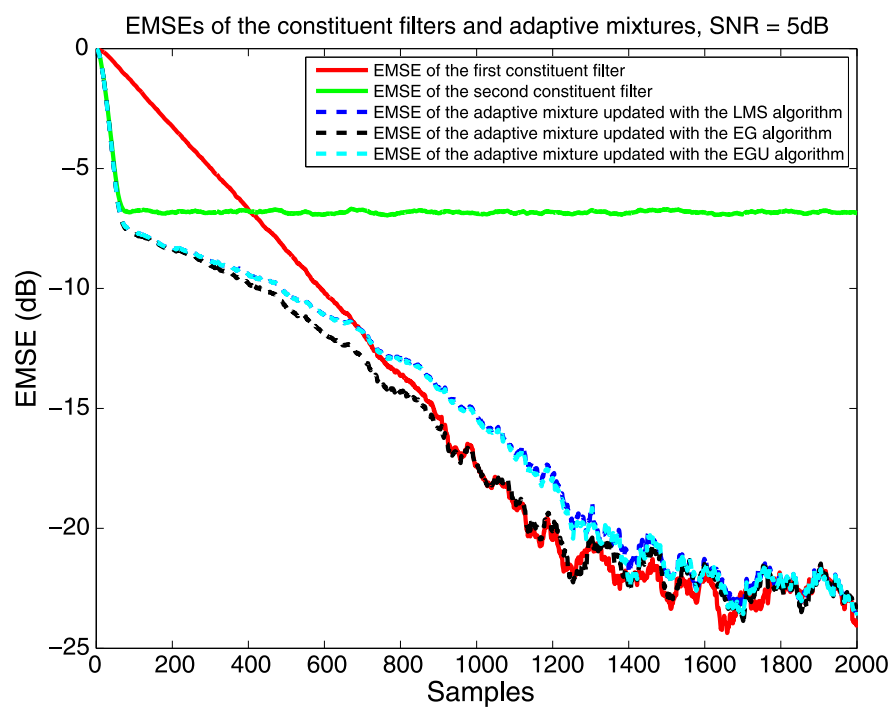

(b)

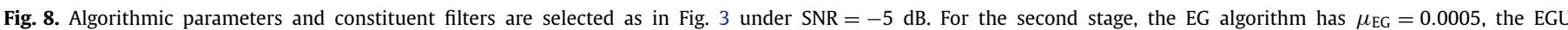

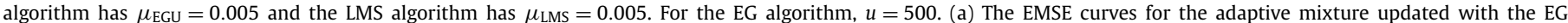

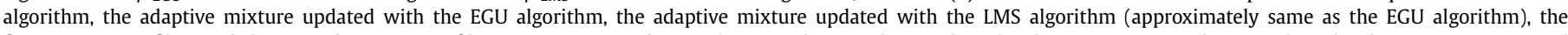

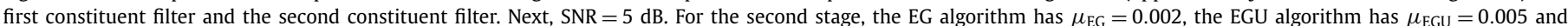

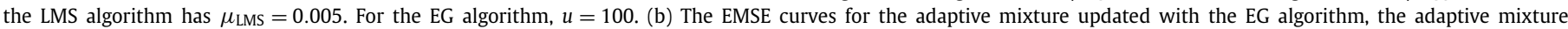

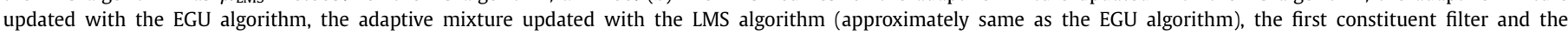
second constituent filter.

a wide range of values for the step sizes so that the algorithms converge to very similar EMSEs. We choose $u=500$ for the EG algorithm. In Fig. 8(a), we plot the EMSE curves for the adaptive mixture updated with the EG algorithm, the adaptive mixture updated with the EGU algorithm, the adaptive mixture updated with the LMS algorithm, first constituent filter with $\mu_{1}=0.002$ and second constituent filter with $\mu_{2} \in[0.1,0.11]$ under $\mathrm{SNR}=-5$. Under $\mathrm{SNR}=5$, learning rates for the EG, EGU and LMS algorithms are selected as $\mu_{\mathrm{EG}}=0.002, \mu_{\mathrm{EGU}}=0.005$ and $\mu_{\mathrm{LMS}}=0.005$. We choose $u=100$ for the EG algorithm. In Fig. 8(b), we plot same EMSE curves as in Fig. 8(a). We observe that the EG algorithm performs better than the EGU and LMS algorithms such that EMSE of the adaptive mixture updated with the EG algorithm converges faster than the EMSE of adaptive mixtures updated with the EGU and LMS algorithms. We also observe that the EGU and LMS algorithms show similar performances when they are used to train the mixture weights.

\section{Conclusion}

In this paper, we investigate adaptive mixture methods based on Bregman divergences combining outputs of $m$ adaptive filters 
to model a desired signal. We use the unnormalized relative entropy and relative entropy as distance measures that produce the exponentiated gradient update with unnormalized weights (EGU) and the exponentiated gradient update with positive and negative weights (EG) to train the mixture weights under the affine constraints or without any constraints. We provide the transient analysis of these methods updated with the EGU and EG algorithms. In our simulations, we compare performances of the EG, EGU and LMS algorithms and observe that the EG algorithm performs better than the EGU and LMS algorithms when the combination vector in steady state is sparse. We observe that the EGU and LMS algorithms show similar performance when they are used to train the mixture weights. We also observe a close agreement between the simulations and our theoretical results.

\section{References}

[1] C. Boukis, D. Mandic, A.G. Constantinides, A class of stochastic gradient algorithms with exponentiated error cost functions, Digital Signal Process. 19 (2009) 201-212.

[2] D.P. Helmbold, R.E. Schapire, Y. Singer, M.K. Warmuth, A comparison of new and old algorithms for a mixture estimation problem, Mach. Learn. 27 (1997) 97-119.

[3] J.C.M. Bermudez, N.J. Bershad, J.Y. Tourneret, Stochastic analysis of an error power ratio scheme applied to the affine combination of two LMS adaptive filters, Signal Process. 91 (2011) 2615-2622.

[4] J. Arenas-Garcia, M. Martinez-Ramon, A. Navia-Vazquez, A.R. Figueiras-Vidal Plant identification via adaptive combination of transversal filters, Signal Process. 86 (2006) 2430-2438.

[5] S.S. Kozat, A.C. Singer, Multi-stage adaptive signal processing algorithms, in: Proceedings of SAM Signal Proc. Workshop, 2000, pp. 380-384.

[6] J. Arenas-Garcia, V. Gomez-Verdejo, M. Martinez-Ramon, A.R. Figueiras-Vidal Separate-variable adaptive combination of LMS adaptive filters for plant identification, in: Proc. of the 13th IEEE Int. Workshop Neural Networks Signal Processing, 2003, pp. 239-248.

[7] J. Arenas-Garcia, M. Martinez-Ramon, V. Gomez-Verdejo, A.R. Figueiras-Vidal, Multiple plant identifier via adaptive LMS convex combination, in: Proc. of the IEEE Int. Symp. Intel. Signal Processing, 2003, pp. 137-142.

[8] J. Arenas-Garcia, V. Gomez-Verdejo, A.R. Figueiras-Vidal, New algorithms for improved adaptive convex combination of LMS transversal filters, IEEE Trans. Instrum. Meas. 54 (2005) 2239-2249.

[9] J. Kivinen, M. Warmuth, Exponentiated gradient versus gradient descent for linear predictors, Inform. Comput. 132 (1997) 1-64.

[10] D.P. Helmbold, R.E. Schapire, Y. Singer, M.K. Warmuth, On-line portfolio selection using multiplicative updates, Math. Finance 8 (4) (1998) 325-347.

[11] N.J. Bershad, J.C.M. Bermudez, J. Tourneret, An affine combination of two LMS adaptive filters: Transient mean-square analysis, IEEE Trans. Signal Process. 56 (5) (2008) 1853-1864.

[12] S.S. Kozat, A.T. Erdogan, A.C. Singer, A.H. Sayed, Steady state MSE performance analysis of mixture approaches to adaptive filtering, IEEE Trans. Signal Process. 58 (2010) 4421-4427.

[13] J. Benesty, Y.A. Huang, The LMS, PNLMS, and exponentiated gradient algorithms, in: Proc. Eur. Signal Process. Conf. (EUSIPCO), 2004, pp. 721-724.

[14] A.H. Sayed, Fundamentals of Adaptive Filtering, John Wiley and Sons, 2003.

[15] J. Arenas-Garcia, A.R. Figueiras-Vidal, A.H. Sayed, Mean-square performance of a convex combination of two adaptive filters, IEEE Trans. Signal Process. 54 (2006) 1078-1090

[16] V.H. Nascimento, M.T.M. Silva, J. Arenas-Garcia, A transient analysis for the convex combination of adaptive filters, IEEE Trans. Signal Process. 58 (8) (2009) 4064-4078.

[17] S.S. Kozat, A.T. Erdogan, A.C. Singer, A.H. Sayed, Transient analysis of adaptive affine combinations, IEEE Trans. Signal Process. 59 (12) (2011) 6227-6232.
[18] V. Vovk, A game of prediction with expert advice, J. Comput. System Sci. 56 (1998) 153-173.

[19] P.A. Naylor, J. Cui, M. Brookes, Adaptive algorithms for sparse echo cancellation, Signal Process. 86 (2006) 1182-1192.

[20] N. Cesa-Bianchi, Y. Freund, D. Haussler, D.P. Helmbold, R.E. Schapire, M.K. Warmuth, How to use expert advice, J. ACM 44 (3) (1997) 427-485.

[21] B. Jelfs, D.P. Mandic, S.C. Douglas, An adaptive approach for the identification of improper complex signals, Signal Process. 92 (2012) 335-344.

[22] D.N. Joanes, C.A. Gill, Comparing measures of sample skewness and kurtosis, J. R. Stat. Soc., Ser. D Stat. 47 (1998) 183-189.

[23] R.A. Fisher, The moments of the distribution for normal samples of measures of departure from normality, Proc. R. Soc. Lond. A 130 (1930) 16-28.

Mehmet Ali Donmez received the B.S. degrees from both the Department of Electrical and Electronics Engineering and the Department of Mathematics, Bogazici University, Turkey, with honors. He is currently a M.S. student at the Department of Electrical and Electronics Engineering of Koc University under the supervision of Professor Suleyman S. Kozat. His current research interests are adaptive signal processing, intelligent systems, online learning, signal processing for communications, signal processing algorithms for mathematical finance and machine learning for signal processing.

Huseyin Atahan Inan received the B.S. degrees from the Department of Electrical and Electronics Engineering and the Department of Mathematics, Bogazici University, Turkey, with a 3.70 GPA. He is currently a M.S. student at the Department of Electrical and Electronics Engineering of Koc University under the supervision of Professor Alper Tunga Erdogan. His current research interests are adaptive signal processing, blind source seperation and independent component analysis and signal processing for communications.

Suleyman Serdar Kozat received the B.S. degree with full scholarship and high honors from Bilkent University, Turkey. He received the M.S. and $\mathrm{Ph} . \mathrm{D}$. degrees in electrical and computer engineering from University of Illinois at Urbana Champaign, Urbana, IL, in 2001 and 2004, respectively.

After graduation, Dr. Kozat joined IBM Research, T.J. Watson Research Center, Yorktown, NY as a Research Staff Member in Pervasive Speech Technologies Group, where he focused on problems related to statistical signal processing and machine learning. While doing his Ph.D., he was also working as a Research Associate at Microsoft Research, Redmond, WA, in Cryptography and Anti-Piracy Group. He holds several patent applications for his works performed in IBM Research and Microsoft Research. After begin an Assistant Professor at Koc University for five years, Dr. Kozat is currently an Assistant Professor at the Electrical and Electronics Engineering Department, Bilkent University, Turkey. Dr. Kozat coauthored more than 50 papers in refereed high impact journals and conference proceedings and has several patent applications. Overall, his research interests include intelligent systems, adaptive filtering for smart data analytics, online learning and machine learning algorithms for signal processing.

Dr. Kozat has been serving as an Associate Editor for the IEEE Transactions on Signal Processing and he is a Senior Member of the IEEE. He has been awarded IBM Faculty Award by IBM Research in 2011, Outstanding Faculty Award by Koc University in 2011, Outstanding Young Researcher Award by the Turkish National Academy of Sciences in 2010, ODTU Professor Dr. Mustafa N. Parlar Research Encouragement Award in 2011 and holds Career Award by the Scientific Research Council of Turkey, 2009. Dr. Kozat has won several scholarships and medals in international and national science and math competitions. 